\title{
Optical constants of refractory oxides at high temperatures ${ }^{\star}$ \\ Mid-infrared properties of corundum, spinel, and $\alpha$-quartz, potential carriers of the $13 \mu \mathrm{m}$ feature
}

\author{
S. Zeidler ${ }^{1}$, Th. Posch ${ }^{2}$, and H. Mutschke ${ }^{1}$ \\ 1 Astrophysikalisches Institut und Universitätssternwarte, Friedrich-Schiller-Universität Jena, Schillergässchen 2-3, \\ 07745 Jena, Germany \\ e-mail: [harald.mutschke;simon.zeidler]@uni-jena.de \\ 2 Institut für Astrophysik, Universität Wien, Türkenschanzstraße 17, 1180 Wien, Austria \\ e-mail: thomas.posch@univie.ac.at
}

Received 28 September 2012 / Accepted 26 February 2013

\begin{abstract}
Context. Many cosmic dust species, among them refractory oxides, form at temperatures higher than $300 \mathrm{~K}$. Nevertheless, most astrophysical studies are based on the room-temperature optical constants of solids, such as corundum and spinel. A more realistic approach is needed for these materials, especially in the context of modeling late-type stars.

Aims. We aimed at deriving sets of optical constants of selected, astrophysically relevant oxide dust species with high melting points. Methods. A high-temperature, high-pressure cell and a Fourier-transform spectrometer were used to measure reflectance spectra of polished samples. For corundum $\left(\alpha-\mathrm{Al}_{2} \mathrm{O}_{3}\right)$, spinel $\left(\mathrm{MgAl}_{2} \mathrm{O}_{4}\right)$, and $\alpha$-quartz $\left(\mathrm{SiO}_{2}\right)$, temperature-dependent optical constants were measured from $300 \mathrm{~K}$ up to more than $900 \mathrm{~K}$. Small particle spectra were also calculated from these data.

Results. All three examined oxides show a significant temperature dependence of their mid-IR bands. For the case of corundum, we find that the $13 \mu \mathrm{m}$ emission feature - seen in the IR spectra of many AGB stars - can very well be assigned to this mineral species. The best fit of the feature is achieved with oblate corundum grains at (mean) temperatures around 550 K. Spinel remains a viable carrier of the $13 \mu$ feature as well, but only for $T<300 \mathrm{~K}$ and nearly spherical grain shapes. Under such circumstances, spinel grains may also account for the $31.8 \mu \mathrm{m}$ band that is frequently seen in sources of the $13 \mu \mathrm{m}$ feature and which has not yet been identified with certainty.
\end{abstract}

Key words. circumstellar matter - infrared: stars - methods: laboratory

\section{Introduction}

Within the past two decades, astromineralogy - the science of solids in space - has progressed significantly. Satellites such as the Infrared Space Observatory (ISO), Spitzer, and Herschel have made it possible to identify emission and absorption bands of crystalline solids in comets, in protoplanetary disks, in the shells of evolved stars, in supernova remnants, and in other environments including quasars (cf. Molste et al. 2010). At the same time, the theory of formation and evolution of minerals in accretion disks and stellar outflows has contributed a lot to our understanding of the gas-solid transition in the cosmos (e.g., Gail 2010). Systematic studies of analog materials in terrestrial laboratories made it possible to establish databases of optical constants of solids that are expected or known to form from gases with a solar composition (e.g. Henning et al. 1999; Henning 2010). These databases continue to be indispensable sources of information on the UV to far-infrared properties of minerals and amorphous solids.

The present paper deals with refractory oxides, i.e. oxide minerals with high melting points. More specifically, we discuss the mid-infrared spectra of corundum $\left(\alpha-\mathrm{Al}_{2} \mathrm{O}_{3}\right)$, spinel

\footnotetext{
* Optical constants are only available at the CDS via anonymous ftp to cdsarc.u-strasbg. fr $(130.79 .128 .5)$ or via http: //cdsarc.u-strasbg.fr/viz-bin/qcat?J/A+A/553/A81
}

$\left(\mathrm{MgAl}_{2} \mathrm{O}_{4}\right)$, and $\alpha$-quartz $\left(\alpha-\mathrm{SiO}_{2}\right)^{1}$ measured at high temperatures (up to $973 \mathrm{~K}$ ). Corundum, spinel, and quartz have repeatedly been proposed as carriers of infrared (IR) bands seen in astronomical objects (see below and Sect. 5). Owing to the lack of more comprehensive data, room temperature dielectric functions, and/or powder transmission, spectra of these minerals have been used in most previous papers. However, the formation of refractory oxides at high temperatures - and the fact that these oxides will partly radiate at high temperatures and leave their spectral fingerprints in their high temperature state - led us to the conclusion that their dielectric functions need to be derived by in situ high-T-measurements. This term is used to denote measurements of materials in their hot state, which is not the same as annealing experiments. In the latter case, a sample is heated to a certain temperature for a while. It may also have undergone an irreversible structural transition, and is afterwards cooled down to room temperature, at which point the spectra are then measured (see, e.g., Jäger et al. 2011, for this distinction).

Thus, what we present here - and for the first time in a systematic way - are in situ high-T-measurements of the IR spectra of $\alpha-\mathrm{Al}_{2} \mathrm{O}_{3}, \mathrm{MgAl}_{2} \mathrm{O}_{4}$, and $\alpha-\mathrm{SiO}_{2}$, taking the effects of anisotropy into account where necessary. Results of annealing

\footnotetext{
1 Note that $\mathrm{SiO}_{2}$ and its polymorphs are sometimes classified as silicates because of their structural similarity to tectosilicates (see e.g. Klein et al. 1999). Nevertheless, we will follow the widespread classification of quartz as an oxide here, following Strunz \& Nickel (2001).
} 
experiments with spinel can be found in Fabian et al. (2001). The optical constants derived in the present paper will be made publicly available ${ }^{2}$.

This paper is structured in the following way: Sect. 2 describes the experimental methods and the samples that have been used. In Sect. 3, we derive temperature-dependent oscillator parameters from our reflectance measurements. Section 4 presents small-particle spectra for different grain shapes. In the final Sect. 5, we discuss astrophysical applications of our data, focusing on the $13 \mu \mathrm{m}$ emission feature in AGB stars.

\section{Experimental methods}

\subsection{The high-temperature, high-pressure cell}

For all our measurements, we used an IR Fourier-transformspectrometer (Bruker 113v) equipped with a water-cooled, hightemperature, high-pressure (HTHP) cell. With this cell (Specac $\mathrm{P} / \mathrm{N} 5850$ ), it is possible to heat samples to temperatures up to $1073 \mathrm{~K}$ and to take IR spectra at the same time. Because of its dimensions, we had to deploy the cell into an aluminum tank that is placed in one of the two sample chambers of the Bruker spectrometer. The tank separates the cell environment, which is flooded with argon gas at atmospheric pressure, from the vacuum inside the spectrometer. The IR beam still can be applied for measurements by using two opposite windows in the tank. We used both potassium bromide $(\mathrm{KBr})$ and polyethylene $(\mathrm{PE})$ windows for measurements in the mid-IR $(3-25 \mu \mathrm{m})$ and far-IR $(25-50 \mu \mathrm{m})$ wavelength ranges, respectively.

The HTHP cell, set in reflectance mode, has been placed into the tank, where a base plate with two adjustable mirrors for the incoming and outgoing IR beam is used (see Fig. 1). The incident angle of the incoming beam on the surface of a sample in the cell is $\sim 12^{\circ}$. We estimate the relative error in reflectance to the case of perpendicular irradiation to be $\sim 3 \%$. The cell itself has a closed sample chamber. The sample holder inside the cell is covered by the heater and has a diameter of $13 \mathrm{~mm}$ and a length of $\sim 15 \mathrm{~mm}$. Special rings can be screwed into the holder to carry and/or to fix the sample.

The HTHP cell is supplied by an external temperature controller by which the heating temperature and the temperature gradient can be set. The controller measures the temperature of the sample holder and the body of the cell. We additionally measured the temperature of the sample holder with an NiCr-Ni thermocouple. The temperatures, thus externally measured, are only by a few percentage points lower than the temperatures measured by the cell. All sample temperatures in this paper are externally measured ones.

\subsection{Samples and sample preparation}

Understanding the changes taking place in refractory oxides at high temperatures requires basic information on their internal structure. Therefore, we give crystallographic information on the respective solids at the beginning of each of the following sections, starting with $\mathrm{Al}_{2} \mathrm{O}_{3}$, the most refractory mineral treated here.

\subsubsection{Corundum}

The distribution of $\mathrm{Al}$ cations in either a face-centered cubic or a hexagonal close-packed lattice of oxygen anions results in

\footnotetext{
2 http://www.astro.uni - jena.de/Laboratory/Database/ databases.html and at the CDS.
}

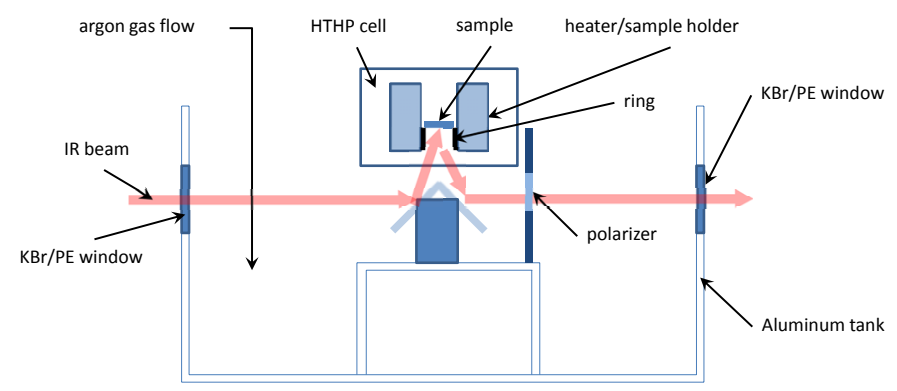

Fig. 1. Sketch of the set up of the HTHP cell in reflection mode in the aluminum tank.

a manifold of polymorphs of $\mathrm{Al}_{2} \mathrm{O}_{3}$. When heated, all polymorphs undergo phase transitions toward the thermodynamically most stable form, namely corundum (also $\alpha-\mathrm{Al}_{2} \mathrm{O}_{3}$; Levin \& Brandon 1998). Corundum has a rhombohedral lattice structure. The oxygen anions are arranged in a (slightly distorted) hexagonal close-packing in which the aluminum cations occupy two thirds of the octahedral interstices. Due to the crystal structure, the interaction with light is not isotropic. The optical constants $n$ and $k$ (real and imaginary part of the complex refractive index) have to be calculated for polarizations perpendicular (ordinary) and parallel (extraordinary) to the crystal's symmetry (c) axis.

Corundum is a highly refractory material. Its condensation temperature is much higher than for most other minerals of astrophysical relevance. At pressures of $\sim 10^{-9}$ bar which occur in stellar outflows of evolved stars, corundum is already stable at more than $1400 \mathrm{~K}$, while silicates condense primarily below $1100 \mathrm{~K}$ (Gail 2010). As a result, corundum should be one of the first condensates to form in the envelopes of $M$ type stars. However, quantum mechanical calculations of the condensation rates have shown that corundum cannot form under these conditions by homogeneous condensation from the gas phase. Gail \& Sedlmayr (1998) point out that the formation of $\mathrm{Al}_{2} \mathrm{O}_{3}$ grains is more likely to occur via grain-surface-reactions on already existing grain seeds of other high refractory materials such as $\mathrm{TiO}_{2}$ or $\mathrm{ZrO}_{2}$.

For the measurements discussed in this paper, we used a plane-parallel disk of pure synthetic corundum, which was manufactured by Thorlabs. This disk has polished surfaces and a diameter of $12.7 \mathrm{~mm}$, whereas the thickness is $3 \mathrm{~mm}$. Temperature-dependent reflectivity measurements on such pure corundum samples have been presented by Gervais \& Piriou (1974a) for temperatures up to $1775 \mathrm{~K}$. In their paper, Gervais $\&$ Piriou present a description of the four-parameter semi quantum (FPSQ) model and a comparison with the classical oscillator model. They show that the FPSQ model is a more effective way to fit the reflectivity of polar crystals in the case of wide reflection bands where equal damping of the longitudinal optical (LO) and transverse optical (TO) mode cannot be expected any more. On the other hand, Thomas et al. (1998a) developed a model that does not need a four-parameter description of the dielectric function but is based on adding temperature-dependent multiphonon contributions to a classical oscillator model. Thomas et al. list the parameters for the oscillators, together with their temperature dependence, corresponding to a second-order polynomial fit. They compared their model with measured data from several other authors and the FPSQ model. An application of the results of Thomas et al. to measured emission spectra of corundum in the wavelength range of $2.5-20 \mu \mathrm{m}$ is presented in the work 
of Sova et al. (1998). However, the presented measurements on corundum in all of these papers refer only to the ordinary ray. To the authors' knowledge, high-temperature IR reflection spectra for the extraordinary ray that are necessary to calculate small particle spectra have not been measured yet. In this paper we present for the first time high-temperature IR reflection measurements of corundum for the ordinary and extraordinary ray and the calculated optical constants based on the FPSQ model.

\subsubsection{Spinel}

In a wider sense, spinels are minerals with the general composition $\left(\mathrm{A}_{1-x} \mathrm{~B}_{x}\right)\left[\mathrm{A}_{x} \mathrm{~B}_{2-x}\right] \mathrm{O}_{4}$. The atoms in parenthesis occupy the tetrahedral sites, and the atoms in square brackets occupy the octahedral sites of a cubic close-packed oxygen lattice. The sum formula of pure $\mathrm{Mg}$-Al-spinel is $\mathrm{MgAl}_{2} \mathrm{O}_{4}$. We will only refer to a material with this stoichiometry as spinel in the following. Spinel crystals with this composition have an elementary cell consisting of 32 oxygen anions, 16 aluminum and 8 magnesium cations. In a perfect spinel crystal, the trivalent Al cations are octahedrally coordinated by oxygen ions, while the bivalent $\mathrm{Mg}$ cations are tetrahedrally coordinated. However, it has been noted by Tropf \& Thomas (1991) and shown in detail by Fabian et al. (2001) that annealing induces the $\mathrm{Mg}$ and $\mathrm{Al}$ ions to change their sites, such that a part of the $\mathrm{Al}$ ions is occupying the tetrahedral sites and a part of the $\mathrm{Mg}$ ions is obversely located in the octahedral sites. This structural transition takes place at $1023-1073 \mathrm{~K}$ and is apparently irreversible at least at short time scales (Tropf \& Thomas 1991; Fabian et al. 2001). Therefore, synthetic spinel crystals are in particular characterized by this deviation from the perfect crystal, since high temperature conditions are needed for their production. In contrast, natural spinel crystals that were exposed to low-temperature conditions for long periods show an almost perfect spinel crystal structure. Apparently, under low temperatures and after long periods, the aluminum cations leave the occupied tetrahedral sites and move to the octahedral sites, while the opposite is true for the magnesium cations (Fabian et al. 2001). However, in the limiting case with zero $\mathrm{Mg}$ content, three eighths of the $\mathrm{Al}$ cations occupy tetrahedral sites, and the spinel transforms into $\gamma-\mathrm{Al}_{2} \mathrm{O}_{3}$.

Spinel has a very high melting point of $\sim 2300 \mathrm{~K}$ at atmospheric pressure (Tropf \& Thomas 1991) - even higher than for terrestrial corundum. However, at the pressures prevailing in the dust-formation zones of AGB-stars (in the range of $\sim 10^{-8} \mathrm{bar}$ ), corundum is able to form first condensates at higher temperatures (at $\sim 1400 \mathrm{~K}$ ) than spinel (which we expect to condense below $1150 \mathrm{~K}$ ). As mentioned above, spinel has a cubic crystal structure and is therefore optically isotropic. In our measurements we used a (pure) synthetic spinel disk (manufactured by SurfaceNet) with a diameter of $12.7 \mathrm{~mm}$ and a thickness of $0.1 \mathrm{~mm}$. Like the corundum disk the spinel sample has polished surfaces. High-temperature data have already been obtained by Thomas et al. (1998a) and by Sova et al. (1998). Thomas et al. published absorption coefficients in the range of $2.5-10 \mu \mathrm{m}$ for various temperatures up to $2000 \mathrm{~K}$ and a comparison of the results with the multiphonon model. Sova et al. measured the emissivity of a pure spinel disk over a wavelength range of $2-12.5 \mu \mathrm{m}$ at $\sim 2000 \mathrm{~K}$ and compared their results with the combined model of multiphonon contributions to the classical dielectric function from Thomas et al. (1998b). Our data cover the whole wavelength range from $5 \mu \mathrm{m}$ to $50 \mu \mathrm{m}$.

\subsubsection{Quartz}

Quartz is one of the three low-pressure phases of crystalline $\mathrm{SiO}_{2}$. The other two are tridymite and cristobalite ${ }^{3}$. It can be divided into a lower temperature, lower symmetry $(\alpha)$ and a higher temperature, higher symmetry $(\beta)$ modification.

The mineral $\alpha$-quartz has a trigonal lattice structure at which the unit cell includes three silicon and six oxygen ions. The silicon cations are tetrahedrally coordinated by four oxygen anions which creates the $\left[\mathrm{SiO}_{4}\right]^{4-}$-tetrahedron, the basic structure of almost all silicates and silica polymorphs. Each oxygen anion belongs to two tetrahedra, which leads to a three-dimensional grid of interconnected tetrahedra, with triples of them winding about a hypothetical axis that is parallel to the c-axis. Thereby, a helix-like structure is formed. It should be mentioned that these structures have no particular influence on the chemical and physical behavior of the crystal, they are just a help in visualizing the basic structure of $\alpha$-quartz.

The change between $\alpha$ - and $\beta$-quartz is taking place at $846-847 \mathrm{~K}$ at atmospheric pressure by a displacive phase transition (Lakshtanov et al. 2007). $\beta$-quartz has a hexagonal lattice structure and its tetrahedra are arrangeded almost in the same way as for $\alpha$-quartz. The only difference lies in the alignment of the tetrahedra. In this respect, $\beta$-quartz has a higher symmetry than $\alpha$-quartz. By cooling down a $\beta$-quartz crystal, the opposite transition occurs, and an $\alpha$-quartz is formed again.

Quartz has been the topic of many studies, since it is one of the most important minerals on Earth and, with its physical and chemical properties, an outstanding material in science and technological applications. Spitzer \& Kleinman (1961) were the first to examine the IR reflection properties of $\alpha$-quartz with respect to classical dispersion theory. They presented very comprehensive room-temperature studies of natural and synthetic quartz crystals. Gervais \& Piriou (1975) presented the first temperaturedependent IR reflection data of quartz. They fit their data between 295 and $975 \mathrm{~K}$ with the already mentioned FPSQ model (Gervais \& Piriou 1974a) and analyzed the changes in the band parameters with temperature, especially in the range of the phase transition from $\alpha$ - to $\beta$-quartz.

In the present study, we took IR reflection measurements on a natural $\alpha$-quartz crystal from Brazil (see Fig. 2). The crystal has a maximum length, height, and width of $11,7.5$, and $8 \mathrm{~mm}$, respectively. EDX analysis did not reveal any case of impurities or deviations from stoichiometry. Because of the crystal structure of $\alpha$-quartz, the interaction with light is anisotropic. As in the case of corundum, one has to distinguish between polarizations parallel and perpendicular to the c-axis. Fortunately, because of the distinct hexagonal shape of the natural crystal used, we could locate the direction of the crystallographic c-axis quite easily. Moreover, the crystallographic c-axis lies parallel to the large natural grown surfaces of our $\alpha$-quartz crystal. Thus, spectroscopic measurements parallel and perpendicular to the c-axis could be taken on one of these surfaces. The crystal was cut $(10 \mathrm{~mm} \times 5 \mathrm{~mm})$ so that it fit into the HTHP cell where the surface used for the measurements has been polished with diamond paste of $0.25 \mu \mathrm{m}$ fineness.

\subsection{Reflectance measurements}

We performed IR measurements with the HTHP cell in reflection mode (see Sect. 2.1) in a wavelength range of 5-50 $\mu \mathrm{m}$. As

3 The high-pressure phases of $\mathrm{SiO}_{2}$ are coesite, stishovite, and seifertite. In total there are ten $\mathrm{SiO}_{2}$ polymorphs (Klein et al. 1999; El Goresy et al. 2008). 


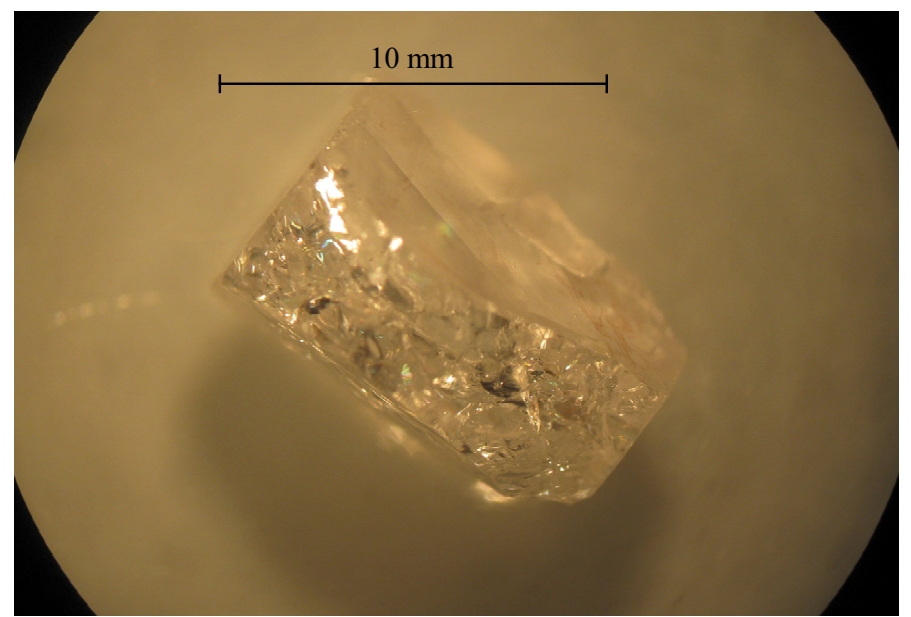

Fig. 2. Photograph of the quartz crystal that has been used for the measurements. The diameter of the field of view is $25 \mathrm{~mm}$.

mentioned above, special rings were used to hold the samples. There are two kinds of these rings: one with a hole of $12 \mathrm{~mm}$ that was used to hold the synthetic samples and one with a hole of $5 \mathrm{~mm}$ for the quartz crystal. For corundum and quartz, a polarizer was placed in the outgoing beam to distinguish between the different crystal orientations. We used two kinds of polarizers: a KRS-5 polarizer for mid-IR measurements $(5-25 \mu \mathrm{m})$ and a PE polarizer for far-IR measurements $(25-50 \mu \mathrm{m})$. With the aid of a low-resolution, real-time spectra shooting in the setup mode of the spectrometer software, the correct polarizer adjustment was established by finding the maximum of bands or by the disappearance of other bands.

As reference for each measurement, a spectrum of a gold mirror with a diameter of $12.7 \mathrm{~mm}$, taken at room temperature ( $300 \mathrm{~K})$, was used. Additionally, IR reflection spectra were taken after the measurements from the empty cell only equipped with the ring that was used for the respective sample. These empty measurements were also taken at the respective temperature of the measured sample and were necessary to exclude influences of the whole sample holder setup. The spectra of sample $R_{\mathrm{S}}$ and empty cell $R_{\mathrm{E}}$, both related to the reference, define the pure reflection spectra $(R)$ via

$R=\frac{R_{\mathrm{S}}-R_{\mathrm{E}}}{1-R_{\mathrm{E}}}$

It could be verified that the heated samples and the sample holders themselves serve as additional IR sources whose emissions contribute to the spectra. While the radiation directly emitted toward the detectors gives a DC signal and is filtered out, the radiation back to the interferometer of the spectrometer becomes modulated and is superimposed on the modulated IR beam from the globar source of the spectrometer. Therefore, all interferograms would actually be superpositions of two sources. To get rid of the additional influence, we measured the interferogram of the heated cell alone by decoupling the globar source and subtracted the result from each interferogram with activated globar source. After this, the resulting interferogram is Fouriertransformed into the desired (single channel) reflection spectrum that is used to calculate $R_{\mathrm{S}}$ or $R_{\mathrm{E}}$.

For the heating process, we used a constant temperature gradient of $10 \mathrm{~K} / \mathrm{min}$ to reach the respective measurement temperature. For corundum and spinel, we took mid-IR spectra at $300 \mathrm{~K}$, $551 \mathrm{~K}, 738 \mathrm{~K}$, and $928 \mathrm{~K}$. For quartz, we also measured at $833 \mathrm{~K}$ to derive more information on the development of the spectrum in the vicinity of the phase transition from $\alpha$ - to $\beta$-quartz. We chose no more than these individual temperatures to prevent the heater from stronger abrasion.

\section{Derived data and data analysis}

\subsection{Derivation of the optical constants}

To calculate the optical constants from reflection measurements, we fitted our data with the FPSQ model of the dielectric function. This model provides the opportunity to fit measured data with independent damping parameters for the LO and the TO mode, while the classical oscillator model only allows to assign different frequencies to the above mentioned two modes. The necessity of assuming independent damping parameters arises in crystals with a strong mode splitting, where LO and TO phonon modes should have different phonon decay times (Gervais \& Piriou 1974a).

Based on the classical dispersion theory, the dielectric function can be written as:

$\epsilon=\epsilon^{\prime}+i \epsilon^{\prime \prime}=\epsilon_{\infty}+\sum_{j} \Delta \epsilon_{j} \frac{\Omega_{j}^{2}}{\Omega_{j}^{2}-\omega^{2}-i \gamma_{j} \omega}$,

where $\Delta \epsilon_{j}, \Omega_{j}$, and $\gamma_{j}$ are the strength, resonance frequency, and damping of the $j$ th oscillator. According to Maxwell's equations, the transverse modes are the poles of the dielectric function, and in contrast the longitudinal modes are the zeros. Barker (1964) has shown that by multiplying all resonant denominators to Eq. (2), a polynomial of degree $2 n$ is created ( $n$ is the number of oscillators) and that this polynomial can be factorized with the zeros of the dielectric function. Consequently, the dielectric function is extended with the LO frequency and damping parameters, and it can be rewritten in its alternative form:

$\epsilon=\epsilon_{\infty} \prod_{j} \frac{\Omega_{j \mathrm{LO}}^{2}-\omega^{2}-i \gamma_{j \mathrm{LO}} \omega}{\Omega_{j \mathrm{TO}}^{2}-\omega^{2}-i \gamma_{j \mathrm{TO}} \omega}$.

This is the FPSQ model in its simplest representation. A deeper treatment of this problem leads to the quantum form of the dielectric function (Gervais \& Piriou 1974a) that goes beyond the classical model toward a quantum mechanical description of the dispersion theory of anharmonic crystals. In our relatively simple case of fitting reflection data at high temperatures, the quantum form will not be important, though it becomes important in discussing the temperature dependence of the mode frequencies and the dampings, so it is rudimentarily discussed in Sect. 3.2.

The optical constants $n$ and $k$ (the real and the imaginary part of the refraction index) are a function of $\epsilon$ represented by the formulae:

$n=\sqrt{\frac{\epsilon^{\prime}}{2}+\frac{1}{2} \sqrt{\epsilon^{\prime 2}+\epsilon^{\prime \prime 2}}}$

$k=\frac{\epsilon^{\prime \prime}}{2 n}$.

The reflection $R$ on a surface at near normal incidence in a vacuum can be expressed as a function of $n$ and $k$ :

$R=\frac{(1-n)^{2}+k^{2}}{(1+n)^{2}+k^{2}}$

Together with Eqs. (3)-(5) measured IR reflection spectra can be fit with the five parameters of this formula.

For all data, the fit procedure has been performed by using the datafit-function of the software SCILAB 5.4.0 (SCILAB consortium 2012). 


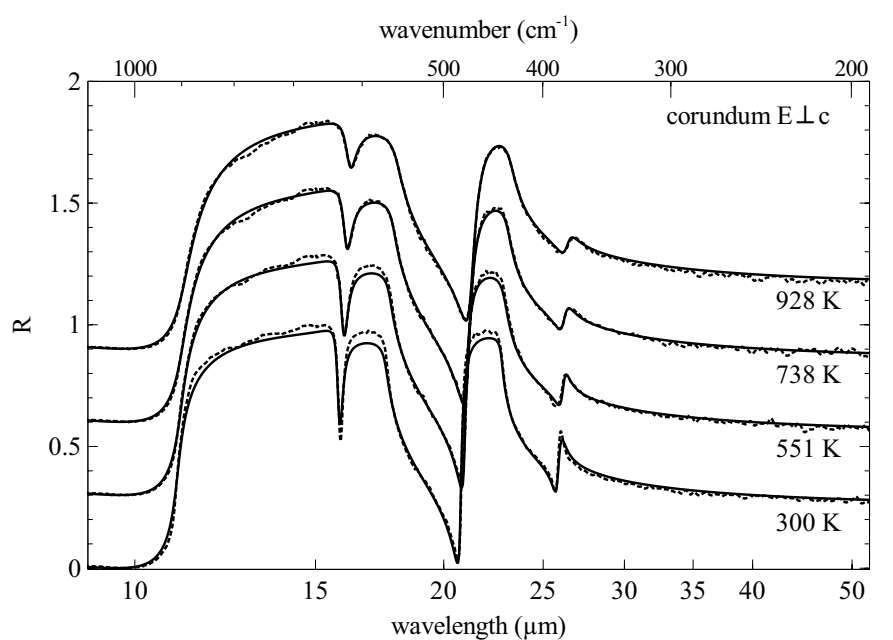

Fig. 3. Comparison of the fittings and the reflection spectra for the ordinary ray ( $E_{u}$-type modes) of the corundum sample at four different temperatures. The fittings have been done with four oscillators.

\subsubsection{Crystalline $\alpha-\mathrm{Al}_{2} \mathrm{O}_{3}$ (corundum)}

In Figs. 3 and 4 the measured reflectivities of the corundum sample for the ordinary and the extraordinary ray at four different temperatures are shown, together with their best fit, with the aid of the FPSQ-model (Eq. (3)). As can be seen, the main changes due to heating occur in the band positions and the intensity of the bands. In general, the bands tend to shift toward longer wavelengths, while the intensity decreases with increasing temperature. This effect is due to the anharmonicity of the atomic potentials and can be seen more clearly in the values of the oscillator parameters listed in Tables 1 and 2. Here, the mode positions move toward longer wavelengths (lower wavenumbers, respectively), and the respective damping constants increase with increasing temperature.

According to the prediction of the irreducible representation of the vibration modes of corundum (Iishi 1978), the spectra for the ordinary ray have been fit with four oscillators ( $E_{u}$-type modes). In contrast, spectra for the extraordinary ray have been fitted with three oscillators, although theory predicts only two oscillators to be IR active ( $A_{2 u}$-type modes). Though, the third oscillator is needed to fit the distinct sharp minimum in the reflection spectra around $21 \mu \mathrm{m}$. An additional band in that range might indicate effects of surface polishing or of imperfect polarization.

The quality of the fits is different, depending on temperature and wavelength range. In general, the noise and the influence of water vapor in the FIR is much stronger than in the MIR, hence the fitting performance, especially for weak bands, is lower, and the quality of the resulting fit decreases for higher temperatures. As can be also seen in Figs. 3 and 4, the maxima of the measured reflectance spectra were not fit exactly. The measured values are either somewhat too high (for $E_{u}$-type modes) or too low (for $A_{2 u}$-type modes). Barker (1964) has shown that the kind of surface treatment can have a strong influence on the measured reflectance spectra profiles owing to additionally excited forbidden modes. Especially the structures on top of the strongest bands measured in both polarization directions give rise to the statement that the differences between measurement and fit in that range are caused by these forbidden modes. Likewise, these structures become weaker with increasing temperature.

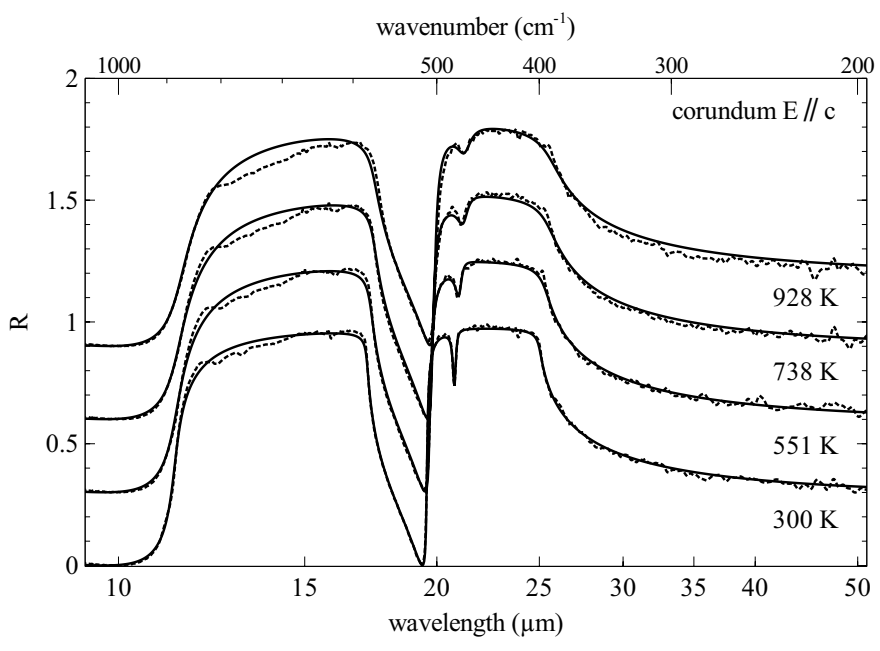

Fig. 4. Comparison of the fittings and the reflection spectra for the extraordinary ray ( $A_{2 u}$-type modes) of the corundum sample at four different temperatures. The fittings have been done with three oscillators.

Table 1. Comparison of the fitting parameters of corundum for the $E_{u}$-type modes $(\mathrm{E} \perp \mathrm{c})$ at different temperatures.

\begin{tabular}{ccccccc}
\hline \hline$j$ & $T(\mathrm{~K})$ & $\begin{array}{c}\Omega_{\mathrm{TO} j} \\
\left(\mathrm{~cm}^{-1}\right)\end{array}$ & $\begin{array}{c}\gamma_{\mathrm{TO} j} \\
\left(\mathrm{~cm}^{-1}\right)\end{array}$ & $\begin{array}{c}\Omega_{\mathrm{LO} j} \\
\left(\mathrm{~cm}^{-1}\right)\end{array}$ & $\begin{array}{c}\gamma_{\mathrm{LO} j} \\
\left(\mathrm{~cm}^{-1}\right)\end{array}$ & $\epsilon_{\infty}$ \\
\hline 1 & 300 & 384.02 & 6.03 & 387.46 & 5.18 & 3.05 \\
& 551 & 380.83 & 6.13 & 383.22 & 6.55 & 3.06 \\
& 738 & 379.9 & 9.86 & 382.36 & 8.56 & 3.08 \\
& 928 & 377.51 & 10.97 & 379.8 & 10.1 & 3.1 \\
& & & & & & \\
2 & 300 & 439.22 & 3.23 & 481.96 & 2.96 & - \\
& 551 & 436 & 7.51 & 477.49 & 3.86 & \\
& 738 & 432.94 & 8.19 & 474.39 & 6.99 & \\
& 928 & 430.21 & 10.49 & 471.18 & 10.15 & \\
3 & & & & & & \\
& 300 & 569.35 & 7.86 & 908.23 & 22.37 & - \\
& 551 & 564.42 & 8.89 & 901.64 & 27.84 & \\
& 738 & 560.1 & 10.24 & 898.5 & 36.78 & \\
& 928 & 555.46 & 14.82 & 894.5 & 45.36 & \\
4 & & & & & & \\
& 300 & 634.36 & 5.6 & 630.59 & 8.53 & - \\
& 551 & 628.8 & 8.13 & 624.95 & 11.37 & \\
& 738 & 624.51 & 10.8 & 620.9 & 14.32 & \\
& 928 & 619.99 & 14.21 & 616.66 & 17.55 & \\
\hline
\end{tabular}

Notes. The definition of the parameters refers to Eq. (3).

\subsubsection{Crystalline $\mathrm{MgAl}_{2} \mathrm{O}_{4}$ (spinel)}

In Fig. 5 the spinel reflection spectra (dotted lines) are compared with their best fit (solid lines) at four different temperatures. The fits are in very good agreement with the measured data and can be treated as almost perfect models for the measurements within the accuracy given by the line thickness.

For a normal, non-defective $\mathrm{MgAl}_{2} \mathrm{O}_{4}$ crystal, group theory predicts only four IR active oscillators whose TO-modes are located at around 15, 18, 21, and $33 \mu \mathrm{m}$ (Thibaudeau 2002). However, the weak band at $43 \mu \mathrm{m}$, as well as the weak shoulders at around 12 and $19 \mu \mathrm{m}$, made it necessary to use eight oscillators for the fitting procedure (see Table 3 ). These additional modes are forbidden by group theory but can be excited by the mentioned disordered cation distribution in synthetic spinels (Fabian et al. 2001) and by defects in the crystal lattice similar to the case of corundum (see above). 
Table 2. Comparison of the fitting parameters of corundum for the $A_{2 u}$-type modes (E\|c) at different temperatures $(j=1,2)$.

\begin{tabular}{ccccccc}
\hline \hline$j$ & $T(\mathrm{~K})$ & $\begin{array}{c}\Omega_{\mathrm{TO}_{j}} \\
\left(\mathrm{~cm}^{-1}\right)\end{array}$ & $\begin{array}{c}\gamma_{\mathrm{TO}} \\
\left(\mathrm{cm}^{-1}\right)\end{array}$ & $\begin{array}{c}\Omega_{\mathrm{LO}_{j}} \\
\left(\mathrm{~cm}^{-1}\right)\end{array}$ & $\begin{array}{c}\gamma_{\mathrm{LO}_{j}} \\
\left(\mathrm{~cm}^{-1}\right)\end{array}$ & $\epsilon_{\infty}$ \\
\hline 1 & 300 & 399.68 & 4.68 & 511.05 & 1.42 & 2.9 \\
& 551 & 394.76 & 11.2 & 508.09 & 2.79 & 2.91 \\
& 738 & 391.91 & 18.58 & 505.24 & 2.88 & 2.91 \\
& 928 & 390.54 & 22.57 & 502.65 & 3.22 & 2.97 \\
& & & & & & \\
2 & 300 & 481.58 & 3.42 & 480.93 & 3.21 & - \\
& 551 & 476.38 & 7.83 & 475.7 & 6.99 & \\
& 738 & 472 & 13.3 & 471 & 12.13 & \\
& 928 & 471.19 & 17.11 & 469.84 & 16.32 & \\
& & & & & & \\
3 & 300 & 582 & 4.17 & 884.75 & 21.57 & - \\
& 551 & 577.36 & 8.93 & 882.32 & 35.96 & \\
& 738 & 573.5 & 11.37 & 878.47 & 44.71 & \\
& 928 & 573.35 & 15.74 & 874.39 & 49.24 & \\
\hline
\end{tabular}

Notes. The third oscillator could not be verified as an $A_{2 u}$-type mode.

Previous studies have also made use of additional modes by fitting reflection spectra of spinel. Fabian et al. (2001) used a classical Lorentz oscillator model and derived fits with eight oscillators in a wavelength range of $9-35 \mu \mathrm{m}$, which means they did not treat the $43 \mu \mathrm{m}$ band. Therefore, they used an extra mode at $11.4 \mu \mathrm{m}$ ( $S$-mode) to fit a weak shoulder on the shortwavelength edge of the broad band between $11-15 \mu \mathrm{m}$, which they link to the $\mathrm{Al} / \mathrm{Mg}$-ratio of their spinel samples and the partial filling of tetrahedral interstices by $\mathrm{Al}$ - instead of Mg-ions. In our spectra we could not see any trace of such a shoulder. Thibaudeau et al. (2002) applied the FPSQ-model to their spectra with six oscillators. Based on their results, we added two more oscillators to the short- and the long-wavelength edges of the broad $19 \mu \mathrm{m}$ band to fit the shoulder at $17 \mu \mathrm{m}$ and the decrement toward longer wavelengths correctly. These extra oscillators have already been found in previous studies (Fabian et al. 2001; O'Horo et al. 1972; Preudhomme \& Tarte 1971) and might be due to the ratio of $\mathrm{Mg} / \mathrm{Al}$-cation disorder and defects, respectively.

Comparing the results of the fitting parameters listed in Table 3, it is noticeable that only the strongest oscillators show a monotone development of their parameters with the temperature. The weaker oscillators $(j=1,3,6)$ do not exhibit such distinct behavior, which might be because in general the fitting of weak modes strongly depends on the quality of the measured spectra, making the fits non-unique (e.g. the weak band around $43 \mu \mathrm{m}$ ). However, in general the mode positions move toward longer wavelengths upon heating, while their respective damping increases with temperature.

\subsubsection{Crystalline $\alpha-\mathrm{SiO}_{2}$ (quartz)}

In contrast to spinel and corundum, the spectra of quartz show a lot of distinct narrow bands, as can be seen in Figs. 6 and 7 for the ordinary and the extraordinary rays, respectively. Theoretical calculations predict eight $E$ - and four $A_{2}$-type modes to be IR active in $\alpha$-quartz, corresponding to the excitations in the ordinary and extraordinary rays, respectively. By contrast, in $\beta$-quartz, four $E$ - and only two $A_{2}$-type modes should be IR active as analyzed by Scott \& Porto (1967). Two $A_{2}$ modes remain from the $\alpha-\beta$-transition of quartz, while the other two become forbidden. The same holds true for the $E$ modes, but here the missing modes

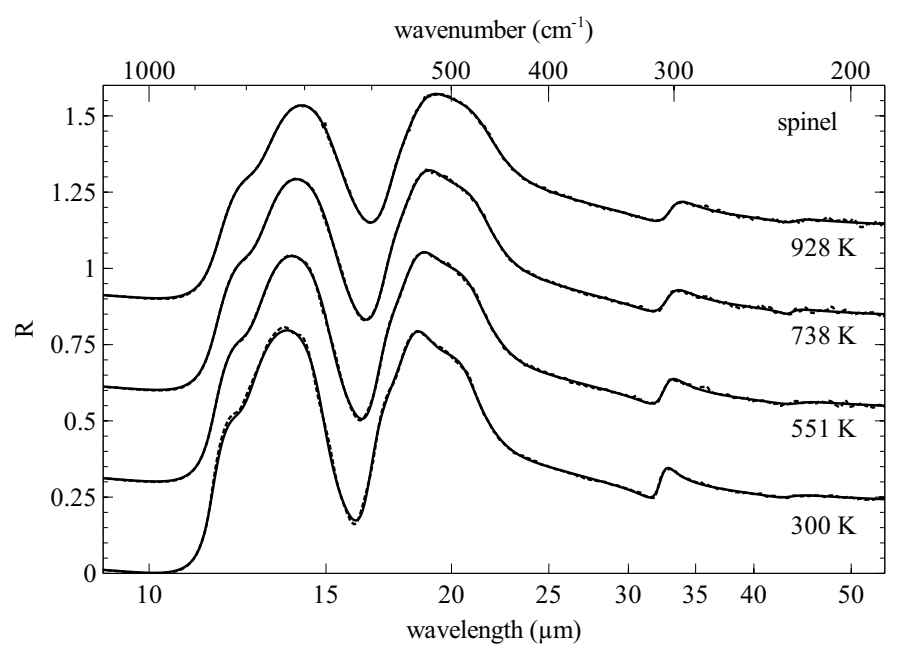

Fig. 5. Reflection spectra of the spinel crystal at four different temperatures.

Table 3. Comparison of the fitting parameters of spinel.

\begin{tabular}{|c|c|c|c|c|c|c|}
\hline$j$ & $T(\mathrm{~K})$ & $\begin{array}{c}\Omega_{\mathrm{TO}_{j}} \\
\left(\mathrm{~cm}^{-1}\right)\end{array}$ & $\begin{array}{c}\gamma_{\mathrm{TO} j} \\
\left(\mathrm{~cm}^{-1}\right)\end{array}$ & $\begin{array}{c}\Omega_{\mathrm{LO} j} \\
\left(\mathrm{~cm}^{-1}\right)\end{array}$ & $\begin{array}{c}\gamma_{\mathrm{LO} j} \\
\left(\mathrm{~cm}^{-1}\right)\end{array}$ & $\epsilon_{\infty}$ \\
\hline \multirow[t]{4}{*}{1} & 300 & 231.05 & 8.97 & 231.58 & 8.29 & 2.77 \\
\hline & 551 & 226.96 & 9.99 & 227.54 & 9.41 & 2.8 \\
\hline & 738 & 230.02 & 9.79 & 230.34 & 9.18 & 2.82 \\
\hline & 928 & 227.33 & 13.81 & 227.78 & 13.45 & 2.77 \\
\hline \multirow[t]{4}{*}{2} & 300 & 307.12 & 12.65 & 310.73 & 13.68 & - \\
\hline & 551 & 304.4 & 16.38 & 308.36 & 17.02 & \\
\hline & 738 & 302.2 & 20.15 & 306.49 & 20.68 & \\
\hline & 928 & 300.07 & 20.15 & 304.01 & 20.58 & \\
\hline \multirow[t]{4}{*}{3} & 300 & 427.11 & 134.26 & 442.85 & 112.26 & - \\
\hline & 551 & 426.34 & 139.54 & 438.44 & 108.65 & \\
\hline & 738 & 438.12 & 140.41 & 444.8 & 96.33 & \\
\hline & 928 & 440.49 & 140.09 & 442.56 & 97.23 & \\
\hline \multirow[t]{4}{*}{4} & 300 & 481.53 & 30.58 & 608.03 & 37.88 & - \\
\hline & 551 & 475.24 & 35.89 & 598.4 & 42.79 & \\
\hline & 738 & 469.66 & 38.7 & 590.55 & 48.62 & \\
\hline & 928 & 466.52 & 45.67 & 582.36 & 55.59 & \\
\hline \multirow[t]{4}{*}{5} & 300 & 531.37 & 55.1 & 558.16 & 70.14 & - \\
\hline & 551 & 527.69 & 63.05 & 553.85 & 74.68 & \\
\hline & 738 & 521.27 & 65.86 & 549.66 & 79.03 & \\
\hline & 928 & 520.32 & 76.53 & 546.93 & 82.07 & \\
\hline \multirow[t]{4}{*}{6} & 300 & 581.8 & 74.64 & 546.32 & 120.96 & - \\
\hline & 551 & 576.95 & 74.12 & 547.55 & 122.79 & \\
\hline & 738 & 569.98 & 75.11 & 542.25 & 126.64 & \\
\hline & 928 & 563.9 & 73.29 & 544.39 & 126 & \\
\hline \multirow[t]{4}{*}{7} & 300 & 672.28 & 36.67 & 866.53 & 39.52 & - \\
\hline & 551 & 662.28 & 47.55 & 862.97 & 47.55 & \\
\hline & 738 & 655.42 & 55.98 & 860.15 & 55.98 & \\
\hline & 928 & 649.32 & 64.24 & 855.98 & 64.24 & \\
\hline \multirow[t]{4}{*}{8} & 300 & 811.63 & 77.37 & 800.67 & 73.77 & - \\
\hline & 551 & 797.84 & 86.36 & 785.72 & 76.12 & \\
\hline & 738 & 789.47 & 92.02 & 776.78 & 79.87 & \\
\hline & 928 & 786.4 & 93.61 & 772.56 & 85.91 & \\
\hline
\end{tabular}

Notes. Oscillators 2, 4, 5, and 7 represent the predicted $T_{1 u}$-type modes of the theory. 


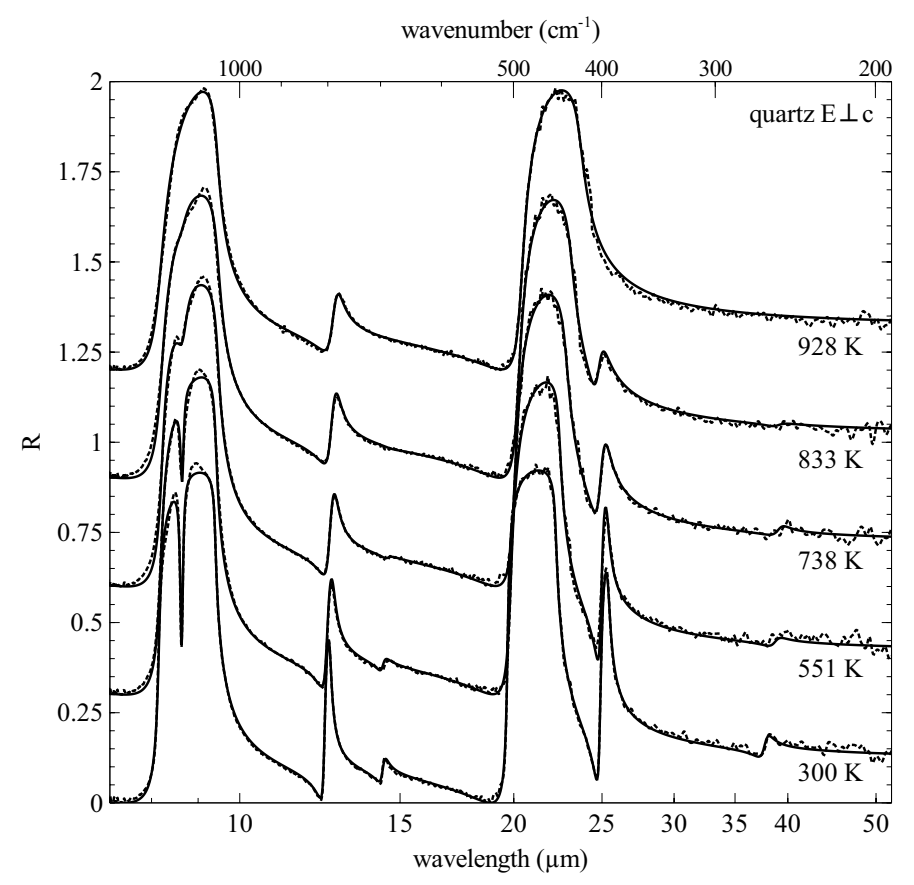

Fig. 6. Reflection spectra of the quartz crystal in the ordinary ray at five different temperatures ( $E$-type modes).

only become IR inactive, while they still remain Raman active (Scott \& Porto 1967).

The fitting parameters for the ordinary and the extraordinary ray at five different temperatures are shown in Tables 4 and 5. We found seven $E$ - and four $A_{2}$-type modes for temperatures below $847 \mathrm{~K}$. The eighth $E$-type mode should be found at wavelengths around $78 \mu \mathrm{m}$, which is, however, beyond our covered wavelength range and therefore has not been measured. Anyway, the parameter values are quite consistent with data given by the literature (Scott \& Porto 1967; Gervais \& Piriou 1975) and they fit our measured data very closely (see Figs. 6 and 7).

By reaching $928 \mathrm{~K}$ for the ordinary ray, it can be clearly seen that the bands at $8.2,14,25$, and $37 \mu \mathrm{m}$ (mode number 7, 4, 2, and 1) disappeared. According to Scott and Porto, these are the four modes that should become IR inactive by the transition to $\beta$-quartz. Already at $833 \mathrm{~K}$, the modes 7,4 , and 1 became very weak so that there is almost no evidence of their existence in the spectrum. In the extraordinary ray, the spectra also show the transition to $\beta$-quartz. Here, modes number 1 and 3 (at 26 and $13 \mu \mathrm{m}$ ) disappeared by reaching $928 \mathrm{~K}$, as predicted by theory. These results correspond very well to the data taken by Gervais \& Piriou (1975), who comprehensively investigated the phase transition of quartz with IR spectroscopy between 7.7 and $33.3 \mu \mathrm{m}$. At this point we can complement their study with data on the temperature development of the $E$-type mode at $37 \mu \mathrm{m}$ that has not been treated in their work.

The quality of the fits decreases at longer wavelengths due to noise and absorption bands of water vapor in the measured spectra. In those with very strong water-absorption bands, we tried to keep the fits slightly above the measured spectra at longer wavelengths since the signal intensity had decreased in that range (see e.g. Fig. 7).

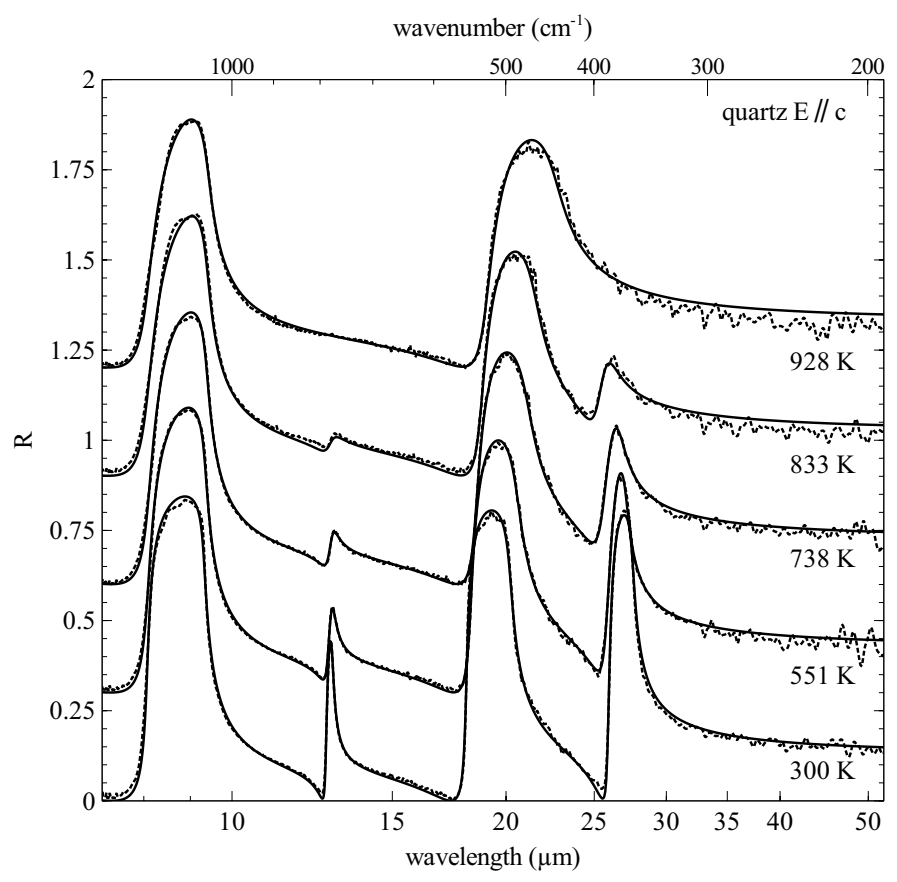

Fig. 7. Reflection spectra of the quartz crystal in the extraordinary ray at five different temperatures ( $A_{2}$-type modes).

\subsection{The temperature dependence of the oscillator parameters}

\subsubsection{Theoretical point of view}

The lattice potential energy of a solid is, in general, anharmonic (Maradudin \& Fein 1962; Cowley 1963). The anharmonicity has, in principle, two main effects on the solid and its spectral properties: thermal expansion of the solid and interactions of phonons of different modes influence their energy and give them a finite lifetime (the reciprocal counterpart to the damping). If the phonon interactions are sufficiently small, the phonon frequency $\Omega_{j}$ for the $j$ th mode as introduced in (3) can be expressed in terms of an unperturbed quasiharmonic mode frequency $\omega_{j}$ and a frequency shift $\Delta \omega_{\mathrm{PI} j}$ representing the influence of the phonon interactions. According to Gervais \& Piriou (1974a,b), $\Omega_{j}$ takes the form

$\Omega_{j}^{2}=\omega_{j}^{2}+2 \omega_{j} \Delta \omega_{\mathrm{PI} j}$

Owing to the anharmonicity, both $\omega_{j}$ and $\Delta \omega_{\mathrm{PI} j},{ }^{4}$ hence $\Omega_{j}$, show a temperature dependence. While for $\Delta \omega_{\mathrm{PI} j}$ the temperature dependence arose from the mode-specific excitation probability of the phonons (Bose-Einstein-distribution), the temperature dependence of $\omega_{j}$ is due to the thermal expansion of the solid and can be approximated by the model of Grüneisen (Lowndes 1970; Jasperse et al. 1966; Gervais \& Piriou 1974a) with:

$\omega_{j}=\omega_{0 j}+\delta \omega_{j}=\omega_{0 j}-\omega_{0 j} \int_{0}^{T} g_{j}\left(T^{\prime}\right) \alpha\left(T^{\prime}\right) \mathrm{d} T^{\prime}$,

where $\omega_{0 j}$ represents the phonon-mode frequency at $0 \mathrm{~K}$ for the $j$ th mode. Here, $g_{j}$ is the respective mode Grüneisen parameter and $\alpha$ the volume thermal expansion of the material. While

4 It should be noted that $\Delta \omega_{\mathrm{PI} j}$ in general carries a frequency dependence that still can be neglected in the FPSQ model (Gervais \& Piriou 1974a). Also, $\Omega_{j}$ is written here as representative of both LO and TO mode frequencies. 
Table 4. Comparison of the fitting parameters of quartz for the ordinary ray.

\begin{tabular}{|c|c|c|c|c|c|c|}
\hline$j$ & $T(\mathrm{~K})$ & $\begin{array}{l}\Omega_{\mathrm{TO} j} \\
\left(\mathrm{~cm}^{-1}\right)\end{array}$ & $\begin{array}{l}\gamma_{\mathrm{TO} j} \\
\left(\mathrm{~cm}^{-1}\right)\end{array}$ & $\begin{array}{l}\Omega_{\mathrm{LO} j} \\
\left(\mathrm{~cm}^{-1}\right)\end{array}$ & $\begin{array}{c}\gamma_{\mathrm{LO} j} \\
\left(\mathrm{~cm}^{-1}\right)\end{array}$ & $\epsilon_{\infty}$ \\
\hline \multirow[t]{5}{*}{1} & 300 & 264.13 & 6.68 & 265.36 & 7.08 & 2.34 \\
\hline & 551 & 257.69 & 9.21 & 258.36 & 9.05 & 2.36 \\
\hline & 738 & 255.06 & 9.29 & 255.77 & 9.87 & 2.38 \\
\hline & 833 & 256.4 & 11.18 & 256.69 & 10.94 & 2.39 \\
\hline & 928 & - & - & - & - & 2.36 \\
\hline \multirow[t]{4}{*}{2} & 300 & 393.55 & 4.25 & 402.88 & 4.33 & - \\
\hline & 551 & 395.03 & 5.26 & 401.96 & 4.88 & \\
\hline & 738 & 396.5 & 10.44 & 402.99 & 11.05 & \\
\hline & 833 & 400.14 & 12.7 & 404.6 & 11.83 & \\
\hline \multirow[t]{5}{*}{3} & 300 & 449.78 & 3.43 & 507.89 & 2.87 & - \\
\hline & 551 & 445.61 & 4.9 & 504.58 & 7.88 & \\
\hline & 738 & 438.27 & 9.28 & 499.84 & 9.29 & \\
\hline & 833 & 432.7 & 11.19 & 495.08 & 10.62 & \\
\hline & 928 & 423.04 & 11.85 & 491.45 & 12.18 & \\
\hline \multirow[t]{4}{*}{4} & 300 & 694.3 & 11.91 & 697.31 & 12.11 & - \\
\hline & 551 & 695.37 & 13.54 & 697.13 & 12.52 & \\
\hline & 738 & 688.59 & 15.17 & 688.99 & 14.51 & \\
\hline & 833 & 680.64 & 38.36 & 680.51 & 37.27 & \\
\hline \multirow[t]{5}{*}{5} & 300 & 795.36 & 7.75 & 808.72 & 8.11 & - \\
\hline & 551 & 790.2 & 13.44 & 804.58 & 13.02 & \\
\hline & 738 & 786.14 & 16.97 & 799.52 & 16.62 & \\
\hline & 833 & 782.98 & 19.92 & 796.81 & 20.2 & \\
\hline & 928 & 780.28 & 22.85 & 793.68 & 26.32 & \\
\hline \multirow[t]{5}{*}{6} & 300 & 1064.57 & 6.98 & 1226.34 & 10.91 & - \\
\hline & 551 & 1063.3 & 9.31 & 1221.61 & 18.66 & \\
\hline & 738 & 1058.36 & 15.17 & 1217.97 & 20.43 & \\
\hline & 833 & 1057.85 & 20.31 & 1220.69 & 31.12 & \\
\hline & 928 & 1060.37 & 19.53 & 1226.38 & 43.43 & \\
\hline \multirow[t]{4}{*}{7} & 300 & 1160.03 & 8.38 & 1157.02 & 7.98 & - \\
\hline & 551 & 1157.62 & 10.57 & 1155.85 & 9.98 & \\
\hline & 738 & 1155.9 & 36.83 & 1153.29 & 34.45 & \\
\hline & 833 & 1154.85 & 41.18 & 1154.19 & 39.93 & \\
\hline
\end{tabular}

for most of the materials $\alpha$ can be approximated well by a linear temperature dependence, $g_{j}$ has a rather complex relation to the temperature (Jasperse et al. 1966), which is often unknown. However, in many cases $g_{j}$ can be assumed as a constant value ranging between 0 and 2 (Lowndes 1970; Gervais \& Piriou 1975), in which case $\delta \omega_{j}$ will show a quadratic temperature dependence.

The phonon-interaction contribution to the frequency shift $\Delta \omega_{\mathrm{PI} j}$ has been determined by Maradudin \& Fein (1962) and by Cowley (1963) involving three phonon processes to secondorder and four phonon processes to first-order pertubation theory. With their assumptions, they obtain a linear temperature dependence of the frequency shift in the high-temperature limit. A quadratic temperature dependence can be obtained by including terms of higher order pertubation theory and/or higher phonon processes as shown by Ipatova et al. (1967) and Gervais \& Piriou (1974b). By assuming $\Delta \omega_{\mathrm{PI} j}$ to be sufficiently small, Eq. (7) can be simplified to

$\Omega_{j}=\omega_{0 j}+\delta \omega_{j}+\Delta \omega_{\mathrm{PI} j}$.

Maradudin \& Fein (1962) pointed out that the frequency shift $\Delta \omega_{\mathrm{PI} j}$ appears as the real part of a complex phonon self energy.
Table 5. Comparison of the fitting parameters of quartz for the extraordinary ray.

\begin{tabular}{ccccccc}
\hline \hline$j$ & $T(\mathrm{~K})$ & $\begin{array}{c}\Omega_{\mathrm{TO}_{j}} \\
\left(\mathrm{~cm}^{-1}\right)\end{array}$ & $\begin{array}{c}\gamma_{\mathrm{TO}_{j}} \\
\left(\mathrm{~cm}^{-1}\right)\end{array}$ & $\begin{array}{c}\Omega_{\mathrm{LO} j} \\
\left(\mathrm{~cm}^{-1}\right)\end{array}$ & $\begin{array}{c}\gamma_{\mathrm{LO}_{j}} \\
\left(\mathrm{~cm}^{-1}\right)\end{array}$ & $\epsilon_{\infty}$ \\
\hline 1 & 300 & 363.03 & 5.18 & 386.42 & 3.76 & 2.39 \\
& 551 & 369.72 & 7.89 & 388.87 & 11.92 & 2.38 \\
& 738 & 376.11 & 15.02 & 392.48 & 18.9 & 2.38 \\
& 833 & 386.59 & 20.1 & 396.64 & 20.05 & 2.38 \\
& 928 & - & - & - & - & 2.44 \\
& & & & & & \\
2 & 300 & 494.99 & 10 & 552.82 & 5.3 & - \\
& 551 & 486.1 & 17.85 & 547.22 & 10.28 & \\
& 738 & 473.93 & 23.83 & 540.15 & 14.12 & \\
& 833 & 465.2 & 24.9 & 533.84 & 17.53 & \\
& 928 & 442.2 & 30.04 & 527.43 & 24.19 & \\
3 & & & & & & \\
& 300 & 775.58 & 8.48 & 789.17 & 6.51 & - \\
& 551 & 775.09 & 13.78 & 785.37 & 15.29 & \\
& 738 & 775.27 & 19.98 & 782.47 & 20.58 & \\
& 833 & 777.02 & 31.45 & 782.29 & 30.85 & \\
4 & & & & & & \\
& 300 & 1073.37 & 15.21 & 1238.42 & 16.41 & - \\
& 551 & 1070.08 & 20.03 & 1237.02 & 30.04 & \\
& 738 & 1066.91 & 22.76 & 1234.71 & 39.73 & \\
& 833 & 1065.91 & 26.52 & 1239.85 & 50.93 & \\
& 928 & 1064.14 & 32.9 & 1240.89 & 55.58 & \\
\hline
\end{tabular}

In their theory, the imaginary part is represented by a function $\Gamma_{j}$ that has the property of altering the phonon energy, so it can be seen as a representative of the damping function. Like $\Delta \omega_{\mathrm{PI} j}$, damping appears as a consequence of the phonon interaction due to anharmonicity, specifically as the inverse of a now finite phonon lifetime. The quantity $\Gamma_{j}$ is related to the classical damping factor $\gamma_{j}$ by the equation

$\gamma_{j}=\Gamma_{j}\left(\frac{2 \omega_{0 j}}{\omega}\right)_{\omega=\Omega_{j}}$.

Like the frequency shift, the damping generally shows a quadratic temperature dependence in the high temperature limit (Ipatova et al. 1967; Gervais \& Piriou 1974b).

\subsubsection{The case of corundum}

The temperature dependence of the complete set of mode parameters for corundum is shown in Fig. 8. For this comparison we chose to plot the relative changes of the parameters with respect to their respective values at $300 \mathrm{~K}$, similar to the low temperature forsterite data presented in Koike et al. (2006). A first look shows that the thermal shift of the resonance frequencies $\Omega_{\mathrm{TO}}$ (upper left panel) is relatively similar for all modes; i.e., the $\Omega_{\mathrm{TO}}$ values change by $1.5 \%-2.5 \%$ for $900 \mathrm{~K}$ relative to $300 \mathrm{~K}$, while for all other parameters describing damping and strength of the resonances the differences among the modes are considerably larger. For some reason, the damping parameters of the $A_{2 u}$-type modes increase more than for the $E_{u}$-type modes, however, no other clear trends (e.g. strong vs. weak modes) can be found.

A fitting of the temperature dependence with respect to the given theory is quite difficult because of the limited temperature range and resolution, especially without any data for lower temperatures. Nonetheless, we try fitting both $\Omega_{j}(T)$ and $\gamma_{j}(T)$ by the quadratic equations

$\Omega_{j}(T)=\Omega_{j}(300)+\beta_{j 1}(T-300)+\beta_{j 2}\left(T^{2}-300^{2}\right)$ 
S. Zeidler et al.: Optical constants of refractory oxides at high temperatures
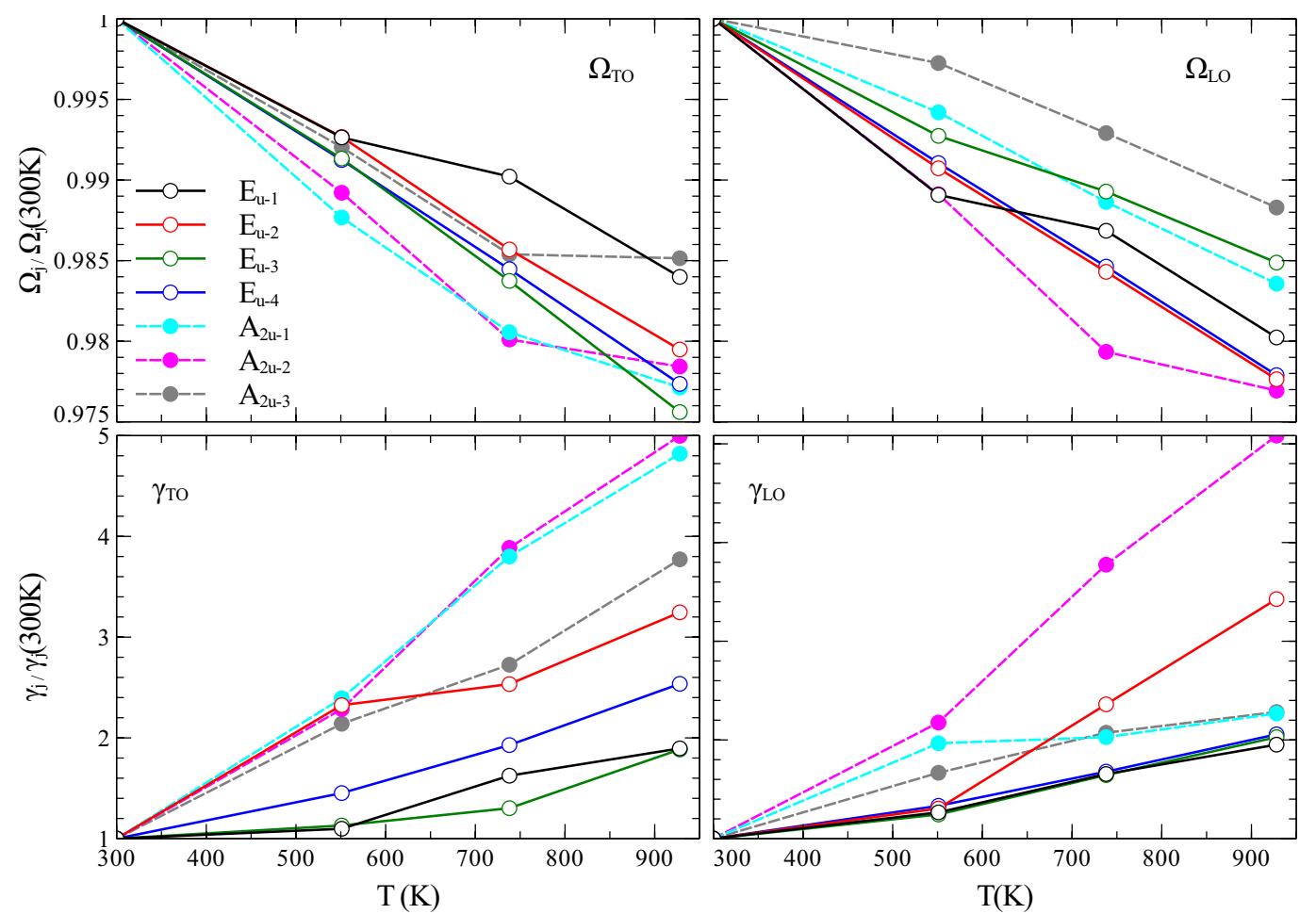

Fig. 8. Comparison of the temperature development of the mode parameters of corundum as given in Tables 1 and 2. The parameters have been normalized to their respective value at $300 \mathrm{~K}$ and are ordered with respect to TO and LO mode, as well as to frequency and damping.

Table 6. Comparison of the temperature-fit parameters of corundum for the modes in the ordinary ray ( $E_{u}$-type modes).

\begin{tabular}{lcccc}
\hline \hline$j$ & $\begin{array}{c}\beta_{j 1} \\
\left(10^{-3} \mathrm{~cm}^{-1} \mathrm{~K}^{-1}\right)\end{array}$ & $\begin{array}{c}\beta_{j 2} \\
\left(10^{-6} \mathrm{~cm}^{-1} \mathrm{~K}^{-2}\right)\end{array}$ & $\begin{array}{c}\gamma_{j 1} \\
\left(10^{-3} \mathrm{~cm}^{-1} \mathrm{~K}^{-1}\right)\end{array}$ & $\begin{array}{c}\gamma_{j 2} \\
\left(10^{-6} \mathrm{~cm}^{-1} \mathrm{~K}^{-2}\right)\end{array}$ \\
\hline 1 (TO) & -9.84 & 0.2 & 0 & 6.6 \\
2 (TO) & -10.67 & -3.1 & 12.02 & 0 \\
3 (TO) & -14.2 & -6.5 & 0 & 7.9 \\
4 (TO) & -19.91 & -2.4 & 2.61 & 9 \\
1 (LO) & -13.62 & 1 & 3.38 & 3.7 \\
2(LO) & -18.39 & 1 & 0 & 8.9 \\
3 (LO) & -23.54 & 1 & 0 & 30 \\
4 (LO) & -22.72 & 0.4 & 6.05 & 6.8 \\
\hline
\end{tabular}

Table 7. Comparison of the temperature fit parameter of corundum for the modes in the extraordinary ray $\left(j=1,3: A_{2 u}\right.$-type modes).

\begin{tabular}{lcccc}
\hline \hline$j$ & $\begin{array}{c}\beta_{j 1} \\
\left(10^{-3} \mathrm{~cm}^{-1} \mathrm{~K}^{-1}\right)\end{array}$ & $\begin{array}{c}\beta_{j 2} \\
\left(10^{-6} \mathrm{~cm}^{-1} \mathrm{~K}^{-2}\right)\end{array}$ & $\begin{array}{c}\gamma_{j 1} \\
\left(10^{-3} \mathrm{~cm}^{-1} \mathrm{~K}^{-1}\right)\end{array}$ & $\begin{array}{c}\gamma_{j 2} \\
\left(10^{-6} \mathrm{~cm}^{-1} \mathrm{~K}^{-2}\right)\end{array}$ \\
\hline 1 (TO) & -17.12 & 1 & 29.21 & 0 \\
2 (TO) & -19.65 & 1 & 15.48 & 5.4 \\
3 (TO) & -17.02 & 1 & 15.53 & 2.1 \\
1 (LO) & -9.34 & -3.4 & 3.25 & 0 \\
2 (LO) & -20.59 & 1 & 8.25 & 10.5 \\
3 (LO) & 3.28 & -16.3 & 47.94 & 0 \\
\hline
\end{tabular}

and

$\gamma_{j}(T)=\gamma_{j}(300)+\gamma_{j 1}(T-300)+\gamma_{j 2}\left(T^{2}-300^{2}\right)$

respectively. In these, we have subtracted the values at $300 \mathrm{~K}$ on both sides, leaving the coefficients $\beta_{j 1}, \beta_{j 2}, \gamma_{j 1}$, and $\gamma_{j 2}$ unchanged with respect to a $0 \mathrm{~K}$-based fitting curve. The coefficients have been restricted such that $\Omega_{j}(T)$ is ensured to monotonically decrease, at least until the melting point of corundum $(\approx 2300 \mathrm{~K})$, while $\gamma_{j}(T)$ has to monotonically increase. Thus, $\beta_{j 1}$ should be normally negative, and $\beta_{j 2}$ is not allowed to be larger than $1 \times 10^{-6} \mathrm{~cm}^{-1} \mathrm{~K}^{-2}$, whereas $\gamma_{j 1}$ and $\gamma_{j 2}$ are both restricted to positive values.

The results are listed in the Tables 6 and 7. As can be seen, only the last $A_{2 u}$-type mode has a positive $\beta_{j 1}$, which is however compensated for by a large negative $\beta_{j 2}$, indicating that the shift of the mode becomes much stronger at high temperature. For some of the $E_{u}$ and most of the $A_{2 u}$-type modes, $\beta_{j 2}$ reaches the positive limit of $1 \times 10^{-6} \mathrm{~cm}^{-1} \mathrm{~K}^{-2}$, which indicates that the data imply a significant weakening of the temperature trend at high $T$. Generally, the absolute value of $\beta_{j 1}$ tends to increase with increasing mode frequency, which is because we fit 
the absolute frequencies here, having already noted above (for the TO frequencies) that the behavior of the relative frequency changes would be similar among the modes.

The behavior of the $\gamma_{j}(T)$ with temperature appears to be quite diverse, as indicated by the fitting parameters. For the $A_{2 u}$-type modes, where the damping is generally rising more sharply with the temperature than for the $E_{u}$-type modes, the changes can more easily be represented by a linear fitting (large $\gamma_{j 1}$ ) and the trend tends to be flattened at higher $T$ (that would result in a negative $\gamma_{j 2}$ but is "zero" due to our restriction). In contrast, for the modes in the ordinary ray ( $E_{u}$-type modes), considerable positive quadratric behavior ( $\gamma_{j 2}$ dominating) is found.

Trying to distinguish between thermal expansion effects and the phonon-interaction contribution in the frequency shift, we write $\Delta \omega_{\mathrm{PI} j}=\Delta_{\mathrm{PI} j 1} T+\Delta_{\mathrm{PI} j 2} T^{2}$ and set $g_{j}$ as temperatureindependent (Eq. (8)). Then, $\beta_{j 1}$ and $\beta_{j 2}$ can be expressed by

$$
\begin{aligned}
& \beta_{j 1}=\Delta_{\mathrm{PI} 1}-\omega_{0 j} g_{j} \alpha_{0} \\
& \beta_{j 2}=\Delta_{\mathrm{PI} j 2}-\omega_{0 j} g_{j} \alpha_{1} / 2,
\end{aligned}
$$

where $\alpha_{0}$ and $\alpha_{1}$ are the coefficients of the linear thermal expansion $\alpha=\alpha_{0}+\alpha_{1} T$. For corundum, they have been determined by Fiquet et al. (1999) to $\alpha_{0}=2.081 \times 10^{-5}$ and $\alpha_{1}=6.6 \times 10^{-9} \mathrm{~K}^{-1}$. At room temperature, Lodziana \& Parlinski (2003) give an interval for all mode Grüneisen parameters of corundum ranging from 0.4 to 1.8 with an averaged value $\langle g\rangle=1.14$. They find that the highest values (1.8) are reached by the $A_{2 u}$ and the lowest $(0.4)$ by the $E_{u}$-type modes. We adopt these values for all temperatures.

Taking the room-temperature resonance frequencies as an estimate of the $\omega_{0 j}$, we can derive, for the latter ( $E_{u}$-type modes, $\left.g_{j}=[0.4,1.14]\right)$, a significant $\Delta_{\mathrm{PI} j 1}$ in the range between $-14.63 \times 10^{-3}$ and $-0.7 \times 10^{-3} \mathrm{~cm}^{-1} \mathrm{~K}^{-1}$. Similar to the mentioned development of $\beta_{j 1}$, the absolute value of $\Delta_{\mathrm{PI} j 1}$ also tends to increase with increasing $\Omega_{j}(300)$. On the other hand, $\Delta_{\mathrm{PI} 2}$ can be positive or negative depending on the mode, and it lies between $-5 \times 10^{-6}$ and $3.09 \times 10^{-6} \mathrm{~cm}^{-1} \mathrm{~K}^{-2}$. It shows no clear development with increasing $\Omega_{j}(300)$.

The estimated values of $\Delta_{\mathrm{PI} j 1}$ for the $A_{2 u}$-type modes in the limits $g_{j}=[1.14,1.8]$ are only for $j=1$ in the same magnitude as the ones for the $E_{u}$-type modes. In the case of $j=3$, they vary between $-3.2 \times 10^{-3}$ and $4.8 \times 10^{-3} \mathrm{~cm}^{-1} \mathrm{~K}^{-1}$. Nevertheless, $\Delta_{\mathrm{PI} j 2}$ is positive for both modes, and it lies between $4 \times 10^{-6}$ and $7.91 \times 10^{-6} \mathrm{~cm}^{-1} \mathrm{~K}^{-2}$. We found an overall increase in the values of both $\Delta_{\mathrm{PI} j 1}$ and $\Delta_{\mathrm{PI} j 2}$ with increasing $\Omega_{j}(300)$. Since both mechanisms (phonon interaction and thermal expansion) generally have negative contributions in their linear coefficients, the calculations confirm that the values of $\beta_{j 1}$ are reasonable.

\subsubsection{Spinel and quartz}

For spinel, the temperature fit is much more difficult due to the weak bands and the unknown degree of ion disorder that can have a big influence on the fits in the case of a synthetic spinel (Thibaudeau et al. 2006). Therefore, because of the missing agreement with the mode parameters, we will not show the temperature fits of spinel.

The temperature dependence of the phonon frequencies and dampings in quartz differs from the assumptions that have been made so far. Quartz already undergos a phase transition at $847 \mathrm{~K}$ that has a strong influence on the behavior of the bands. Gervais $\&$ Piriou (1975) found that in the vicinity of the phase transition the temperature dependence of the frequency shift for $\alpha$-quartz is almost only related to the thermal expansion. Furthermore, the dampings show a linear temperature dependence up to $700 \mathrm{~K}$ as would appear if only cubic anharmonicity were taking place, while a critical increase appears in the approach of the phase transition. In contrast, all bands that survived the phase transition to $\beta$-quartz do not show a strong temperature behavior any longer, even in the vicinity of the transition temperature. This behavior is similar to that of the thermal expansion (Gervais \& Piriou 1975). Another interesting point is the temperature behavior of the frequencies of the modes $(E)_{j=2}$ and $\left(A_{2}\right)_{j=1}$. It shows a monotone increase in both TO and LO vibrations, whereas all other modes show a more ore less smooth decrease. Both bands disappear by reaching the transition point.

The possible temperature development of $\epsilon_{\infty}$ has been neglected in the FPSQ calculations of Gervais \& Piriou (1974a and 1975) for corundum and quartz as well as in those of Thibaudeau et al. (2006) for spinel. However, Cowley (1963) has shown that $\epsilon_{\infty}$ has a temperature dependence when taking multiphonon contributions into account. Therefore, while fitting our data, we allowed $\epsilon_{\infty}$ to vary and could indeed verify a certain temperature dependence for all measured materials, with the general trend toward an increase in $\epsilon_{\infty}$ with increasing temperature. This result is supported by the data of Thomas et al. (1998a), who compared measured near IR refractive indexes of corundum with their multiphonon model. They found a linear increase of $n$ with increasing temperature, corresponding to a quadratic increase of $\epsilon_{\infty}$ (since the absorption is too small to count for $\epsilon$ in that wavelength range). Unfortunately, we cannot give any analytical temperature dependence of $\epsilon_{\infty}$ until we have more data at higher and lower temperatures, which would increase the accuracy of the resulting fit.

\section{Calculation of small-particle spectra}

Using the dielectric function $\epsilon$ (see Eq. (3)) that was calculated during the fitting procedure, we derived the absorption cross section for spherical particles small compared to the wavelength with the aid of the theory by Gustav Mie (Mie 1908). If we let $a$ be the grain radius and $\lambda$ the wavelength, then

$$
C_{\mathrm{abs}}=\frac{8 \pi^{2} a^{3}}{\lambda} \operatorname{Im}\left(\frac{\epsilon-1}{\epsilon+2}\right) .
$$

For nonspherical particle shapes, a useful method for calculating small-particle spectra is the distribution of form factors (DFF) model (Min 2006). In the framework of this model, the absorption cross section is given by a generalization of Eq. (14) in the form

$C_{\mathrm{abs}}=\frac{8 \pi^{2} a^{3}}{\lambda} \int_{0}^{1} \operatorname{Im} \frac{\epsilon-1}{1+L(\epsilon-1)} \frac{P(L)}{3} \mathrm{~d} L$,

where $P(L)$ is a distribution function of form factors $L$ that are defined in the interval $(0 ; 1)$ and contain the information about the particle shape. In this case, $a$ is the radius of a volumeequivalent sphere. For $P(L)=\delta(L=1 / 3)$, Eq. (14) is obtained. Mutschke et al. (2009) have demonstrated that certain synthetic DFFs derived by Min et al. (2006) for aggregates of spherical particles and for Gaussian random sphere particles are well suited to reproduce the measured $300 \mathrm{~K}$ absorption spectra of typical corundum and spinel powders with either more roundish or more irregular grain shapes, respectively. We calculate such absorption spectra at high temperatures in addition to those of spherical particles to demonstrate the diversity of spectra that can be derived from our optical constants. 
S. Zeidler et al.: Optical constants of refractory oxides at high temperatures

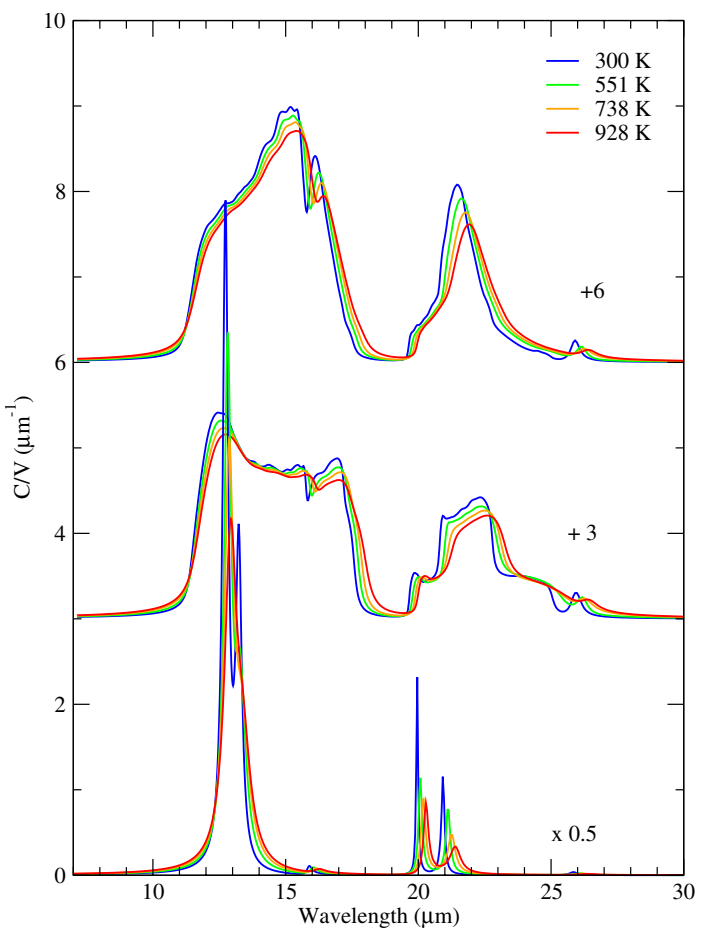

Fig. 9. Comparison of the volume normalized $C_{\mathrm{abs}}$ of small particles of corundum for Mie theory (multiplied by 0.5 ), a DFF model for aggregated spheres (offset +3 ), and a DFF model for Gaussian random spheres $($ offset +6$)$ at different temperatures.

For materials with anisotropic optical behavior like quartz and corundum, the absorption cross section has to be calculated for each incoming-beam polarization parallel to the crystal axes a, b, and c, and the results have to be summarized to an averaged absorption cross section $C_{\text {abs }}$. In the case of corundum and quartz, the polarization parallel to $\mathrm{a}$ and $\mathrm{b}$ results in identical spectra, which means that the absorption cross section for the ordinary ray $\left(C_{\text {abso }}\right)$ has to be taken with double weight compared to the absorption cross section for the extraordinary ray $C_{\text {abs eo }}$ :

$C_{\text {abs }}=\frac{1}{3} C_{\text {abs eo }}+\frac{2}{3} C_{\text {abso }}$.

In the case of spinel, the averaging is of course not necessary. It has to be noted that for all nonspherical particle models, the separate treatment of the crystal axes in calculating the absorption cross sections is physically not exact and can lead to deviations in the predicted spectra from reality. This can only be circumvented by much more complex models such as the discrete dipole approximation. In the main bands of corundum and spinel, however, the deviations are minor, and the spectra shown in the following are sufficiently reliable. For details see Mutschke et al. (2009).

In Figs. 9-11, the calculated temperature-dependent $C_{\text {abs-spectra of corundum, spinel, and quartz are presented. In }}$ each figure, the spectra for small spheres (Mie theory), aggregates of spheres and Gaussian random spheres are compared. For all three minerals, the differences between the spectra of Mie theory (bottom spectra) and those of the DFF model (offset spectra) are the most prominent. In Mie theory, the strongest modes appear as very sharp and intense bands in the spectra of $C_{\text {abs }}$ while already for aggregated spheres (offset +3 ), the bands become much broader toward longer wavelengths, although a maximum remains at the position of the Mie peak. In the spectra of the Gaussian random spheres (offset +6 ), even the maximum of the

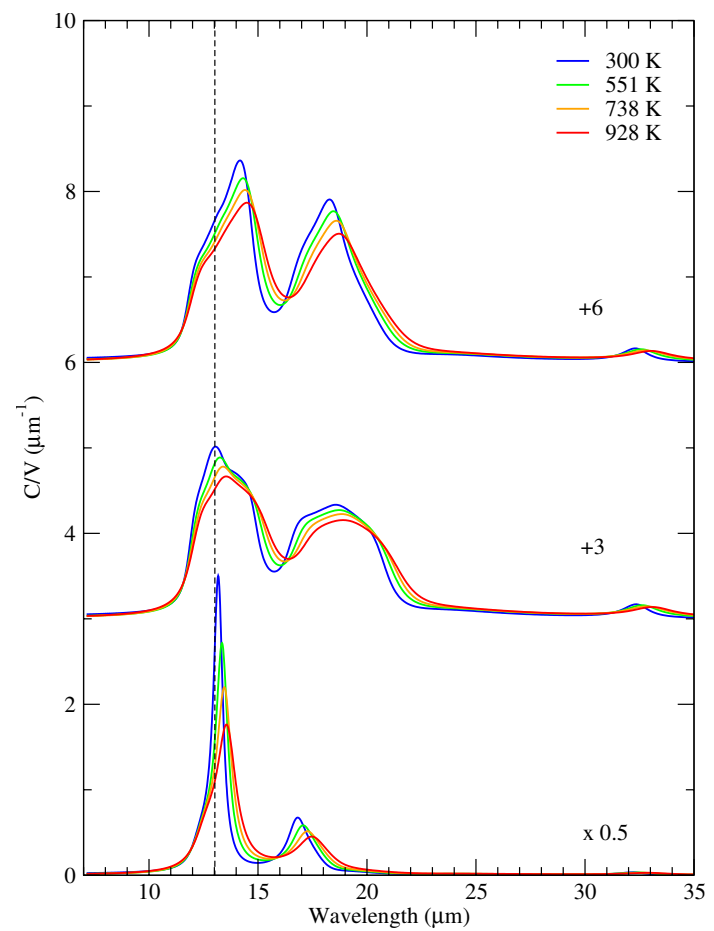

Fig. 10. Comparison of the volume normalized $C_{\text {abs }}$ of small particles of spinel for Mie theory (multiplied by 0.5 ), a DFF model for aggregated spheres (offset +3 ), and a DFF model for Gaussian random spheres $($ offset +6$)$ at different temperatures. The dashed vertical line indicates the $13 \mu \mathrm{m}$ band position.

bands becomes shifted toward longer wavelength, while only a shoulder remains at the position of the Mie peak. Therefore, the FWHM of the bands in the spectra of Gaussian random spheres is not very different to the one of the aggregates of spheres. Note that most changes in the $C_{\mathrm{abs}}$-spectra take place only for the strongest bands. The weaker bands do not show any strong particle shape dependence.

\section{Consequences of our measurements for the astronomical $13 \mu \mathrm{m}$ band}

The small-particle spectra derived in the previous sections enable a better understanding of solid state bands, especially those detected in warm and hot dust-forming astronomical environments. Indeed, the most straightforward application of our laboratory spectra is the onset phase of dust-formation in AGB stars, where mainly minerals belonging to the hot end of the oxygenrich condensation sequence form. This is because our data refer to refractory minerals, and our main innovation is the systematic study of the high temperature spectra of these minerals.

Interestingly, there is a subset of dust-forming stars on the Asymptotic Giant Branch (AGB), where refractory dust species, such as corundum and/or spinel seem to be over-represented compared to the silicates. (The latter tend to be the overwhelmingly dominating kind of dust for other oxygen-rich stars on the AGB.) This subset is characterized by optically thin shells, in most cases by a semiregular pulsational behavior (Sloan et al. 1996), and - in terms of IR spectroscopy - by the presence of the so-called 13 micron feature ${ }^{5}$, which has been the subject of

\footnotetext{
5 About $90 \%$ of the semiregular variables for which IR spectra are available show the $13 \mu \mathrm{m}$ feature according to Sloan et al. (1996).
} 


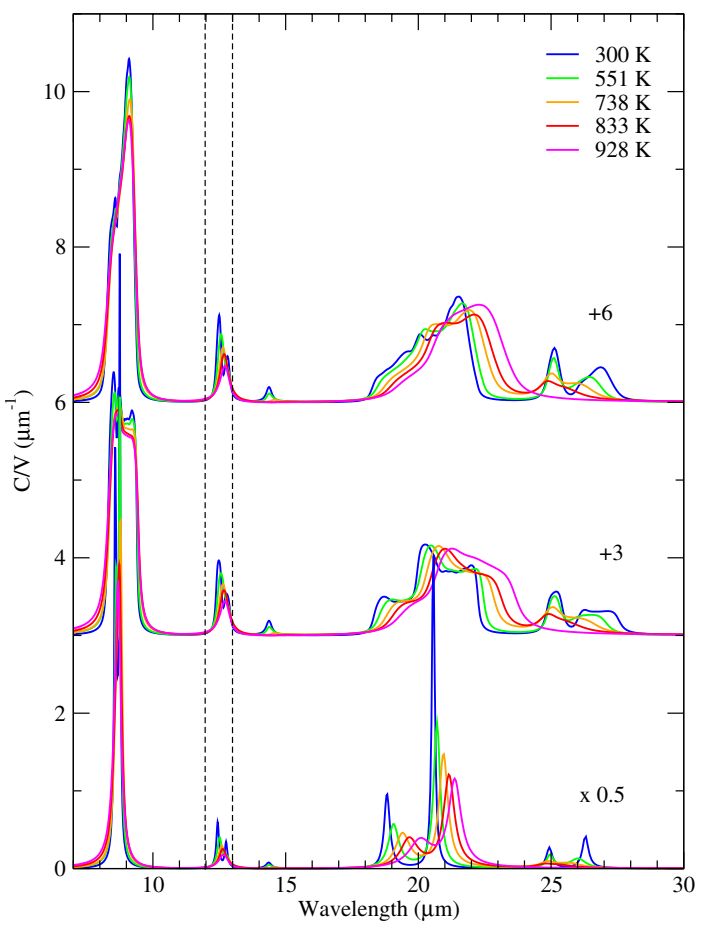

Fig. 11. Comparison of the volume normalized $C_{\mathrm{abs}}$ of small particles of quartz for Mie theory (multiplied by 0.5 ), a DFF model for aggregated spheres (offset +3 ), and a DFF model for Gaussian random spheres (offset +6 ) at different temperatures. The features that could account for the astronomical $13 \mu \mathrm{m}$ band are actually located between 12 and $13 \mu \mathrm{m}$ (hence the dashed vertical lines).

many papers (e.g. Glaccum 1995; Sloan et al. 1996, 2003; Posch et al. 1999; Fabian et al. 2001; DePew et al. 2006), and even of a Ph.D. Thesis (DePew 2006).

Consensus has been reached that this spectral feature is due to an oxide rather than to a silicate dust species. In fact, all three dust species discussed in this paper have been proposed as carrier of the $13 \mu \mathrm{m}$ band. Speck et al. (2000) propose (amorphous) $\mathrm{SiO}_{2}$ as feature carrier, Posch et al. (1999) and Fabian et al. (2001) favor $\mathrm{MgAl}_{2} \mathrm{O}_{4}$, Sloan et al. (2003) and DePew et al. (2006) have strengthened the case for $\alpha-\mathrm{Al}_{2} \mathrm{O}_{3}$. It should be noted that this dust feature has also been detected in AGB stars outside our galaxy (e.g. Lebzelter et al. 2006) and in the spectra of S-type AGB stars (Smolders et al. 2012).

As for $\mathrm{SiO}_{2}$, the optical constants that we measured at high temperatures do not make it a more viable candidate carrier of the $13 \mu \mathrm{m}$ band than the room temperature data did. The by far strongest band of hot $\alpha-\mathrm{SiO}_{2}$ is located at wavelength of $8.7-8.9 \mu \mathrm{m}$ for spherical grains and also remains around $9 \mu \mathrm{m}$ for the nonspherical grain shapes we examined. However, no significant narrow 8.7-9 $\mu \mathrm{m}$ emission feature is detected in those astronomical objects that show the $13 \mu \mathrm{m}$ band. The sole (weak) resonance mode of hot $\alpha-\mathrm{SiO}_{2}$ which comes close to $13 \mu \mathrm{m}$ is in fact located around $12.5-12.7 \mu \mathrm{m}$, with only a weak dependence on the grain shape, as mentioned before and as demonstrated in Fig. 11. As a result, $\alpha-\mathrm{SiO}_{2}$ cannot be considered as a promising candidate carrier of the $13 \mu \mathrm{m}$ band; so we focus on the application of our high temperature data for hot spinel and corundum.

The following procedure has been applied to compare the small-particle spectra derived from our optical constants with astronomical spectra:

i) each $C_{\mathrm{abs}}$ spectrum (for our measured $T_{\mathrm{d}}$ values) is multiplied with the corresponding black body function $B_{v}\left(T_{\mathrm{d}}\right)$, and the result is normalized to unity. We refer to the resulting curves, i.e. to the normalized $C_{\mathrm{abs}} \times B_{v}(T)$ curves, as normalized dust emissivities.

ii) Selected spectra of AGB stars showing the $13 \mu \mathrm{m}$ bands are treated as outlined by Posch et al. (1999) and Fabian et al. (2001) to derive residual dust emissions. A polynomial is used to represent all other dust components but the carrier of the $13 \mu \mathrm{m}$ band. The respective polynomial is then subtracted from the spectra. The result is also normalized to unity. A mean out of 23 such residual dust emissions (based on ISO spectra) was calculated. In short, the following comparisons confront normalized dust emissivities with mean residual dust emissions in the $11-15 \mu \mathrm{m}$ range. Just for comparison, the residual emission spectrum of an S-type AGB star as seen with Spitzer - taken from Smolders et al. (2012) - is also shown.

\subsection{Hot corundum and the $13 \mu \mathrm{m}$ band}

As mentioned before, crystalline $\alpha-\mathrm{Al}_{2} \mathrm{O}_{3}$ has been considered as a potential carrier of the $13 \mu \mathrm{m}$ feature; e.g., Glaccum (1995) wrote that "hot sapphire is the most likely source of the $13 \mu \mathrm{m}$ feature found in some M and MS stars" (but he did not indicate on which IR data this assertion was based). As for the term "sapphire", we will avoid it in the following, since it refers to $\alpha-\mathrm{Al}_{2} \mathrm{O}_{3}$ containing coloring impurities, e.g. small amounts of $\mathrm{Fe}^{2+}, \mathrm{Fe}^{3+}, \mathrm{Ti}^{3+}$ or $\mathrm{V}^{4+}$. We instead refer to pure $\alpha-\mathrm{Al}_{2} \mathrm{O}_{3}$ and use the term corundum for it. Evidence of the formation of $\mathrm{Al}_{2} \mathrm{O}_{3}$ came from extensive research on presolar grains in meteorites (e.g. Nittler 1997; Clayton \& Nittler 2004; Hoppe 2004). However, it has so far not been possible to set any strong constrain on the polytype of $\mathrm{Al}_{2} \mathrm{O}_{3}$ by presolar grain studies (which is a problem, since many polytypes of $\mathrm{Al}_{2} \mathrm{O}_{3}$ exist - see, e.g., Tamanai et al. 2009).

Until very recently, it has been impossible to produce any satisfying fit of the $13 \mu \mathrm{m}$ band using optical constants of corundum at room temperature. As shown by Posch et al. (1999), the following dilemma arose, based on the room temperature data:

a) for small spherical particles, corundum would produce a $12.7 \mu \mathrm{m}$ feature with too narrow a bandwidth;

b) for a continuous distribution of ellipsoids, $\alpha-\mathrm{Al}_{2} \mathrm{O}_{3}$ would produce a $13.2 \mu \mathrm{m}$ feature with an up to 7 times too large bandwidth.

Takigawa et al. (2012) proposed to solving this dilemma by introducing ellipsoidal grains of a particular shape. In their condensation experiments, they found that the condensation rates of crystalline $\mathrm{Al}_{2} \mathrm{O}_{3}$ are quite different for the different crystallographic axes, which leads to a flattening of the condensed particles along the crystallographic c-axis. They derive an axis ratio of $r_{\mathrm{c}} / r_{\mathrm{a}}=0.79$ and note that for oblate spheroidal grains with a slightly lower ratio of $r_{\mathrm{c}} / r_{\mathrm{a}}=0.70$, the position of the $13 \mu \mathrm{m}$ feature could be well reproduced with room temperature optical constants. This is because, compared to spherical grains, such oblate spheroids have a resonance for the $\mathrm{E} \perp \mathrm{c}$ contribution at slightly longer wavelengths, while the minor resonance of the E\|c contribution shifts to shorter wavelengths. This leads to a single peak, which in the case of the experimentally derived axis ratio $r_{\mathrm{c}} / r_{\mathrm{a}}=0.79$ would still be positioned a bit shortward of the $13 \mu \mathrm{m}$ feature and even narrower than the spherical resonance. Allowing for a range of axis ratios around a somewhat changed value of $r_{\mathrm{c}} / r_{\mathrm{a}}=0.70$ would give the right feature (cf. Takigawa et al. 2012; see also Bohren \& Huffman 1983, 


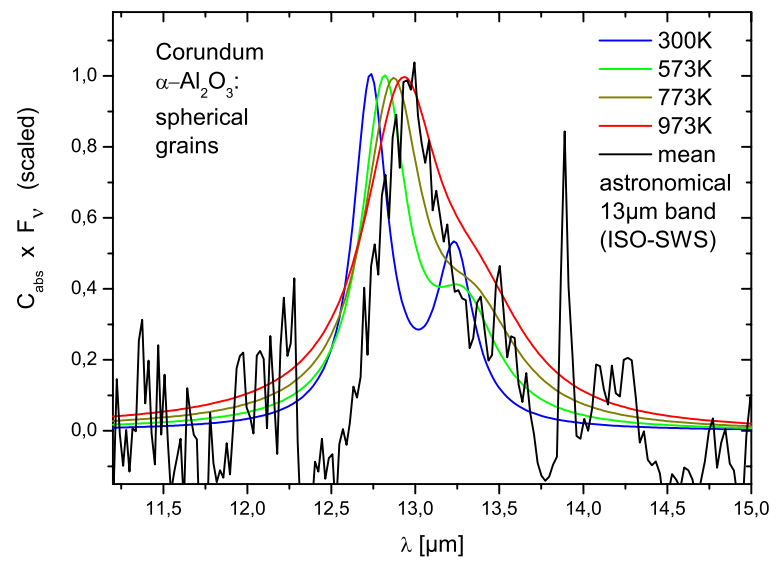

Fig. 12. An average profile of the astronomical $13 \mu \mathrm{m}$ emission band - as derived by Fabian et al. (2001) for a sample of 23 oxygen-rich AGB stars - compared to the normalized emission spectra of spherical corundum grains at temperatures of $300 \mathrm{~K}$ to $928 \mathrm{~K}$.

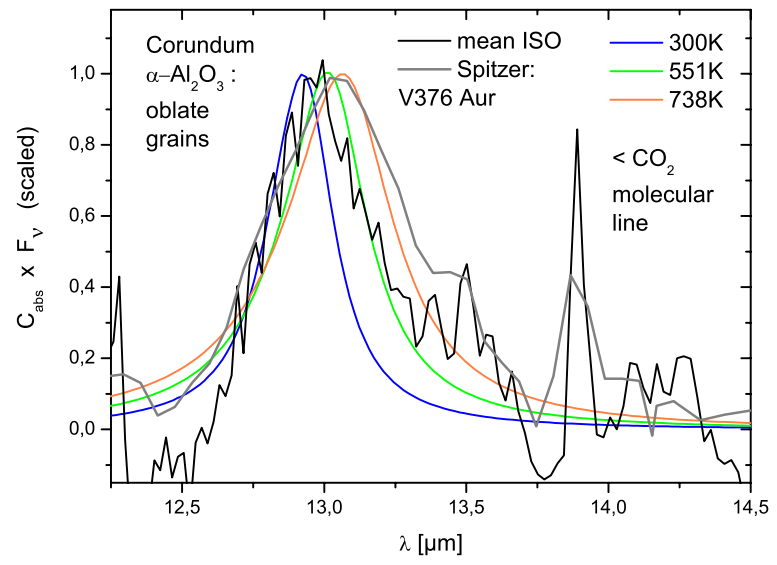

Fig. 13. The case of nonspherical corundum grains. These grains, particularly rotational ellipsoids with an axis ratio of $r_{\mathrm{c}} / r_{\mathrm{a}}=0.79$, enable a good representation of the observed $13 \mu \mathrm{m}$ band profile (the latter is the same as in the previous figure). In addition to our mean ISO $13 \mu \mathrm{m}$ band profile, a single Spitzer-IRS $13 \mu \mathrm{m}$ band profile (of V376 Aur, kindly provided by Smolders) is also shown.

Sect. 5.3, for the applied method of calculating $C_{\text {abs }}$ for ellipsoidal grains). We show in the Figs. 12 and 13 how our hightemperature data partly confirm and partly modify these results.

Figure 12 refers only to spherical grains. The plot shows how the increasing temperature shifts the peak position of the strongest resonance of spherical corundum grains to longer wavelengths, until a position of almost exactly $13 \mu \mathrm{m}$ is reached for temperatures close to $1000 \mathrm{~K}$. However, the bandwidth significantly increases with temperature. At the same time, the peak height $C_{\text {abs,max }}$ is reduced, which is not seen in the normalized spectra. It will be noticed that for $T=928 \mathrm{~K}$, the width of the $13 \mu \mathrm{m}$ corundum band is too large compared to its (average) astronomical counterpart. It should be noted, though, that there are individual astronomical objects with slightly broader $13 \mu \mathrm{m}$ bands than others (cf. Posch et al. 1999; Table 1 and Fabian et al. 2001, Table 4). We also verified this with Spitzer-IRS spectra, kindly provided by K. Smolders. Broader band profiles may indicate higher (mean) dust temperatures.

Figure 13 refers to oblate spheroidal grains with the experimental axis ratio found by Takigawa et al. (2012), i.e. $r_{\mathrm{c}} / r_{\mathrm{a}}=$ 0.79 . With the room temperature data, we find, in agreement with their result, that the calculated band is at a somewhat shorter wavelength and that a further flattening of the particle shape would be necessary for a perfect match. For grains radiating at $T=551 \mathrm{~K}$, however, a very good representation of the observed $13 \mu \mathrm{m}$ band profile is achieved. For still higher temperatures, the corundum peak is shifted even further to the red (see the curve for $T=738 \mathrm{~K}$ in Fig. 13). This leads to a discrepancy with our mean (ISO-SWS-based) profile of the $13 \mu \mathrm{m}$ band (see below).

It should be noted that only one percent of corundum in the circumstellar shell of an AGB star are sufficient to account for the strength of the $13 \mu \mathrm{m}$ band. This has been shown by DePew et al. (2006). In this study, the discrepancy between the peak position of corundum spheres and the observed $13 \mu \mathrm{m}$ band had not become evident due to a relatively coarse wavelength grid (used to speed up the radiative transfer calculations, typically with one grid point every $0.5 \mu \mathrm{m}$ around $13 \mu \mathrm{m}$; see their Fig. 5).

The study by DePew et al. (2006) also reveals how it may be possible that we do see corundum's (or spinel's) $\sim 13 \mu$ m feature, while we do not see their weaker bands at longer wavelengths: those bands may simply be suppressed by temperature effects and owing to the superposition of other dust components (see also the final section of Sloan et al. 2003).

Another argument (other than Fig. 13) for corundum being the carrier of the $13 \mu \mathrm{m}$ band fact that this band is frequently detected together with a broad spectral feature peaking at $\sim 11-12 \mu \mathrm{m}$, which is usually assigned to amorphous $\mathrm{Al}_{2} \mathrm{O}_{3}$. The coexistence of crystalline corundum and amorphous $\mathrm{Al}_{2} \mathrm{O}_{3}$ in an oxygen-rich circumstellar shell with a low mass loss rate seems quite likely. Incomplete condensation of a gas of solar composition might at the same time result in the formation of amorphous alumina and in a relative lack of silicates (compared to shells with greater optical depths that had more time to form silicates as well, in addition to refractory oxides - see, e.g., Smolders et al. 2012 for a short discussion of this idea).

\subsection{Hot spinel and the $13 \mu \mathrm{m}$ band}

For the case of spinel, we restrict ourselves on the small particle spectra of spherical grains - since, as pointed out by DePew et al. (2006), nonspherical grains of spinel can hardly account for the specific properties (bandwidth, bandshape) of the $13 \mu \mathrm{m}$ band. More specifically, a distribution of ellipsoidal grains or hollow spheres leads to a broadening and shift to longer wavelengths of spinel's main MIR emission band, which is incompatible with the properties of the $13 \mu \mathrm{m}$ feature (see also Posch et al. 1999).

In Fig. 14, the same astronomical spectra as in the previous figures are shown in comparison to the high temperature emissivities of spinel grains. It becomes evident from this figure that the $300 \mathrm{~K}$ emissivity of spinel - i.e. its room temperature spectrum! - comes closest to the profile of the $13 \mu \mathrm{m}$ band. For all the higher temperatures, the bandwith of spinel's strongest emission signature becomes much broader than for the astronomical counterpart (with the FWHM reaching $1.38 \mu \mathrm{m}$ at $928 \mathrm{~K}$ ). The peak position shifts to $13.5 \mu \mathrm{m}$ for the largest examined temperature $928 \mathrm{~K}$, which is also at odds with the peak wavelength of the $13 \mu \mathrm{m}$ feature. For nonspherical spinel grains, the $13 \mu \mathrm{m}$ band peak position shifts to even slightly longer wavelengths than $13.5 \mu \mathrm{m}$ (see Fig. 10). Table 8 summarizes essential properties of the observed $13 \mu \mathrm{m}$ band and of its potential carriers (spinel and corundum) radiating at different temperatures.

The peak in the mean ISO spectrum at $16.8 \mu \mathrm{m}$ seems to coincide with a minor emissivity peak of spinel at room temperature, as noted by Posch et al. (1999) and Fabian et al. (2001). However, the $16.8 \mu \mathrm{m}$ band seen in some astronomical 


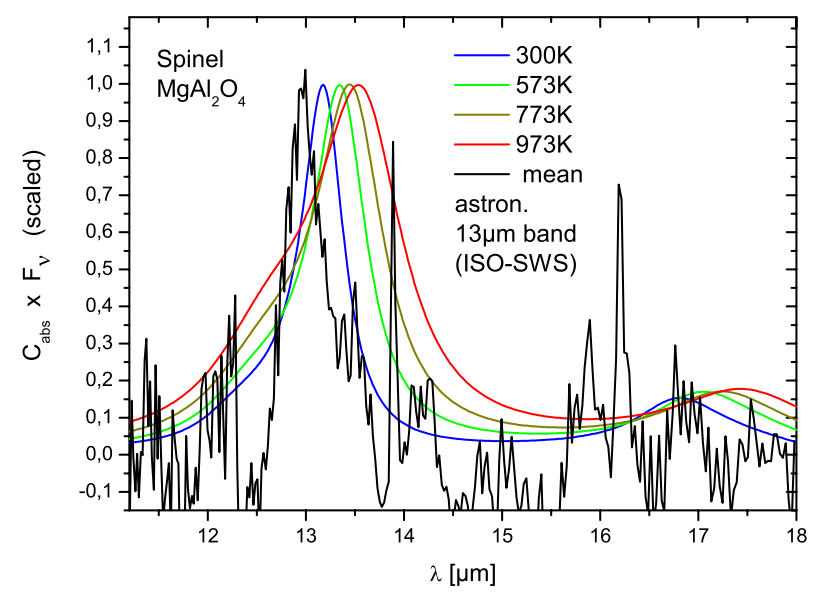

Fig. 14. Comparison between the average profile of the astronomical $13 \mu \mathrm{m}$ emission band - same as in the previous figure - with the normalized emission spectra of spherical spinel grains at temperatures of $300 \mathrm{~K}$ to $928 \mathrm{~K}$.

Table 8. Comparison of the properties of the observed $13 \mu \mathrm{m}$ band profile with those of spinel and corundum at different temperatures, according to our new in situ high temperature optical constants.

\begin{tabular}{lccc}
\hline \hline $\begin{array}{l}\text { Substance } \\
\text { or spectrum }\end{array}$ & $\begin{array}{r}\lambda_{\text {peak }} \\
{[\mu \mathrm{m}]}\end{array}$ & $\begin{array}{c}F W H M \\
{[\mu \mathrm{m}]}\end{array}$ & Comments \\
\hline Mean ISO-SWS & 13.00 & 0.45 & \\
V376 Aur, Spitzer-IRS & 13.07 & 0.60 & \\
corundum, spher., 300 K & 12.73 & 0.26 & + shoulder \\
& & & at $13.2 \mu \mathrm{m}$ \\
corundum, spher., 551 K & 12.82 & 0.35 & \\
corundum, spher., 738 K & 12.87 & 0.50 & \\
corundum, spher., 928 K & 12.94 & 0.73 & \\
corundum, obl., 300 K & 12.92 & 0.27 & \\
corundum, obl., 551 K & 13.00 & 0.37 & \\
corundum, obl., 738 K & 13.07 & 0.46 & \\
spinel, spher., 300 K & 13.17 & 0.53 & \\
spinel, spher., 551 K & 13.35 & 0.72 & \\
spinel, spher., 738 K & 13.44 & 0.97 & \\
spinel, spher., 928 K & 13.53 & 1.37 & \\
\hline
\end{tabular}

sources of the $13 \mu \mathrm{m}$ feature has meanwhile been assigned to a $\mathrm{CO}_{2}$ molecular line (Sloan et al. 2003). Therefore, this small spectral band can no longer be used to support the assignment of the $13 \mu \mathrm{m}$ band to spinel.

There is yet another spectral feature that spinel grains, if present in a circumstellar shell, are supposed to produce, namely an emission band peaking close to $32 \mu \mathrm{m}$ (Fabian et al. 2001). Indeed, there is a narrow emission feature at $31.8 \mu \mathrm{m}$ in many of the sources of the $13 \mu \mathrm{m}$ band. Therefore, we show an average profile of this $31.8 \mu \mathrm{m}$ band (according to Posch et al. 2006) and compare it to the emissivity of hot spinel grains in Fig. 15. This comparison shows that even for spherical grains at room temperature, no satisfying fit of the astronomical $31.8 \mu \mathrm{m}$ band with stoichiometric spinel can be achieved (the bandwidth for spinel at $T=300 \mathrm{~K}$ is too large, and the peak position is at too large a wavelength). For spinel at higher temperatures, our new optical constants lead to an even larger discrepancy between its emissivity and $31.8 \mu \mathrm{m}$ band profile. We rather expect that spinel grains cooler than $300 \mathrm{~K}$ produce a $31.8 \mu \mathrm{m}$ band with the required (narrow) bandwidth, but presently available data do not yet allow a decision on this question.

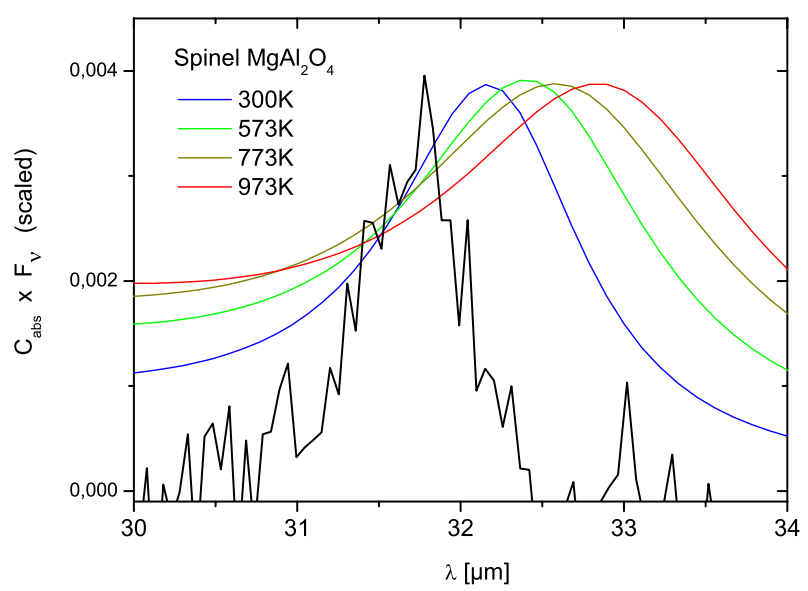

Fig. 15. Comparison between the average profile of the astronomical $32 \mu \mathrm{m}$ emission band with the normalized emission spectra of spherical spinel grains at temperatures of $300 \mathrm{~K}$ to $928 \mathrm{~K}$.

\section{Concluding remarks}

Our new optical constants show that both spinel and corundum grains, if radiating at a suitable narrow range of inermediate temperatures, can account for the average profile of the astronomical $13 \mu \mathrm{m}$ band: spinel at about $300-400 \mathrm{~K}$ and corundum at about 500-700 K (also depending on the grain shapes).

At still higher grain temperatures, the resulting integrated emission, both of spinel and of corundum grains, becomes too broad as to fit the observed astronomical band. The precise temperature at which spinel's and/or corundum's $13 \mu \mathrm{m}$ band becomes too broad and too strongly "redshifted" compared to the observations depends on the individual astronomical object under examination, since some observed $13 \mu \mathrm{m}$ bands of AGB stars are broader and/or peak at longer wavelengths than others. Therefore, the $13 \mu \mathrm{m}$ band may even be used as a dust temperature indicator, at least at a somewhat more advanced stage of astronomical MIR spectrosopy (with even more available MIR spectra of AGB stars and with increased signal-tonoise ratios).

So far, we have fitted only the $13 \mu \mathrm{m}$ band with our new optical constants of hot corundum and spinel. Both of them have additional weaker bands at longer wavelengths for which no counterparts in the observed astronomical spectra could be found so far. For spinel's $32 \mu \mathrm{m}$ feature, there is an astronomical counterpart, but at a smaller peak wavelength and with a narrower bandwidth (only compatible with temperatures of $\mathrm{MgAl}_{2} \mathrm{O}_{4}$ grains below $\left.300 \mathrm{~K}\right)$.

The lack of (some) longer wavelength features may be due to a suppression of those bands by temperature effects, as shown by other authors (e.g. de Pew et al. 2006). Radiative transfer calculations should be made again, based on our optical constants, in order to precisely predict the strengths of those secondary bands (at an expected fractional abundance of corundum and/or spinel amounting to a few percentage points) and to check systematically with which fitting parameters such predictions are indeed compatible with presently available astronomical spectra.

Acknowledgements. We thank Gabriele Born, Jena, for the sample preparation and for help with the EDX measurements. Kristof Smolders, Leuven, kindly provided Spitzer spectra of V376 Aur. Furthermore, we are very thankful to an anonymous referee who helped us improve the paper. This project is a part of the SPP ("Schwerpunktprogramm") of the DFG (Deutsche Forschungsgemeinschaft): "The First 10 Million Years of the Solar System". 


\section{References}

Barker, A. S. 1964, Phys. Rev., 136, 1290

Barker, A. J., Wilkinson, G. R., Massa, N. E., et al. 1975, in Optical Properties of Highly Transparent Solids, (New York: Plenum), eds. S. S. Mitra, \& B. Bendow

Begemann, B., Dorschner, J., Henning, Th., \& Mutschke, H. 1997, ApJ, 476, 199

Bohren, C. F., \& Huffman, D. R. 1983, in Absorption and Scattering of Light by Small Particles (New York: John Wiley)

Burns, R. G. 1993, in Mineralogical Applications of Crystal Field Theory, 2nd edn. (Cambridge University Press), 5

Cami, J. 2002, Ph.D. Thesis, University of Amsterdam

Choi, B.-G., Huss, G. R., Wasserburg, G. J., \& Gallino, R. 1998, Science, 282, 1284

Clayton, D. D., \& Nittler, L. R. 2004, ARAA, 42, 39

Cowley, R. A. 1963, Adv. Phys., 12, 421

DePew, K. 2006, Ph.D. Thesis, University of Missouri-Columbia

DePew, K., Speck, A., \& Dijkstra, C. 2006, ApJ, 640, 971

El Goresy, A., Dera, P., Sharp, T. S., Prewitt, C. T., \& Cheng, M. 2008, Eur. J. Mineral., 20, 523

Fabian, D., Posch, Th., Mutschke, H., Kerschbaum, F., \& Dorschner, J. 2001, A\&A, 373, 1125

Fiquet, G., Richet, P., \& Montagnac, G. 1999, Phys. Chem. Minerals, 27, 103

Gail, H.-P. 2010, in Astromineralogy, ed. Th. Henning (Berlin and Heidelberg: Springer-Verlag), 2nd edn., Lect. Notes Phys., 815, 61

Gail, H.-P., \& Sedlmayr, E. 1998, Faraday Discuss, 109, 303

Gervais, F., \& Piriou, B. 1974a, J. Phys. C: Solid State Phys., 7, 2374

Gervais, F., \& Piriou, B. 1974b, Phys. Rev. B, 10, 1642

Gervais, F., \& Piriou, B. 1975, Phys. Rev. B, 11, 3944

Glaccum, W. 1995, in Airborne Astronomy Symposium on the Galactic Ecosystem, eds. M. R. Haas, J. A. Davidson, \& E. F. Erickson, ASP Conf. Ser., 73, 395

Henning, Th. 2010, in Astromineralogy, ed. Th. Henning (Berlin and Heidelberg: Springer-Verlag), 2nd edn., Lect. Notes Phys., 815, 313

Henning, Th., \& Mutschke, H. 1997, A\&A, 327, 743

Hoppe, P. 2004, in Astrophysics of Dust, ASP Conf. Ser., 309, 265

Huss, G. R., Hutcheon, I. D., Wasserburg, G. J., \& Stone, J. 1992, LPI Contributions, 781, 29

Huss, G. R., Fahley, A. J., Gallino, R., \& Wasserburg, G. J. 1994, ApJ, 430, 81

Iishi, K. 1978, Phys. Chem. Minerals, 3, 1

Ipatova, I. P., Maradudin, A. A., \& Wallis R. F. 1967, Phys. Rev., 155, 882

Jäger, C., Posch, Th., Mutschke, H., et al. 2011, IAU Symp., 280, 416

Jasperse, J. R., Kahan, A., Plendl, J. N., \& Mitra, S. S. 1966, Phys. Rev., 146, 526

Klein, C., Hurlbut, C. S., \& Dana, J. D. 1999, in Manual of Mineralogy (New York: Wiley)

Koike, C., Mutschke, H., Suto, H., et al. 2006, A\&A, 449, 583

Lakshtanov, D. L., Sinogeikin, S. V., \& Bass, J. D. 2007, Phys. Chem. Minerals, 34,11

Lebzelter, Th., Posch, Th., Hinkle, K., Wood, P. R., \& Bouwman, J. 2006, ApJ, 653, L145
Levin, I., \& Brandon, D. 1998, J. Am. Ceram. Soc., 81, 1995

Lodziana, Z., \& Parlinski, K. 2003, Phys. Rev. B, 67, 174106

Lowndes, R. P. 1970, Phys. Rev. B, 1, 2754

Maradudin, A. A., \& Fein, A. E. 1962, Phys. Rev. B, 128, 6

Mie, G. 1908, Ann. Phys., 25, 377

Min, M., Hovenier J. W., Dominik, C., de Koter, A., \& Yurkin, M. A. 2006, J. Quant. Spectrosc. Radiat. Transf., 97, 161

Molster, F. J., Waters, L. B. F. M., \& Kemper, F. 2010, in Astromineralogy, ed. Th. Henning (Berlin and Heidelberg: Springer-Verlag), 2nd edn., Lect. Notes Phys., 815, 143

Mutschke, H., Min, M., \& Tamanai, A. 2009, A\&A, 504, 875

Nittler, L. R. 1997, in Astrophysical Implications of the Laboratory Study of Presolar Materials, eds. T. J. Bernatowicz, \& E. Zinner, AIP Conf. Proc., 402, 59

O’Horo, M. P., Frisillo, A. L., \& White, W. B. 1972, J. Phys. Chem. Solids, 34, 23

Palik E. D. 1985-1998, Handbook of Optical Constants of Solids, 3 vols. (Boston: Academic Press)

Philipp, H. R. 1985, in Handbook of Optical Constants of Solids I, ed. E. D. Palik (Boston: Academic Press), 749

Posch, Th., Kerschbaum, F., Mutschke, H., et al. 1999, A\&A, 352, 609

Posch, Th., Kerschbaum, F., Mutschke, H., et al. 2003, ESA SP, 511, 141

Posch, Th., Mutschke, H., Kerschbaum, F., \& Lebzelter, Th. 2006, Rev. Mod. Astron., 19, 251

Preudhomme, J., \& Tarte, P. 1971, Spectrochim. Acta, 27 A, 1817

Scilab Consortium 2012, Scilab: The free software for numerical computation, http://www.scilab.org

Scott, J. F., \& Porto, S. P. S. 1967, Phys. Rev., 161, 903

Sloan, G. C., LeVan, P. D., Little-Marenin, \& I. R. 1996, ApJ, 463, 310

Sloan, G. C., Kraemer, K. E., Goebel, J. H., \& Price, S. D. 2003, ApJ, 594, 483

Smolders, K., Neyskens, P., Blommaert, J. A. D. L., et al. 2012, A\&A, 540, A72

Sova, R. M., Linevsky, M. J., Thomas, M. E., \& Mark, F. F. 1998, Infrared Phys. Techn., 39, 251

Speck, A. K., Barlow, M. J., Sylvester, R. J., \& Hofmeister, A. M. 2000, A\&AS, 146,437

Spitzer, W. G., \& Kleinman, D. A. 1961, Phys. Rev., 121, 1324

Strunz, H., \& Nickel, E. H. 2001, Strunz Mineralogical Tables, 9th edn. (Stuttgart: Schweizerbart Verlag)

Takigawa, A., Tachibana, S., Nagahara, H., \& Ozawa, K. 2012, LPI Science Conference Abstracts, 43, 1875

Tamanai, A., Mutschke, H., Blum, J., et al. 2009, A\&A, 501, 251

Thibaudeau, P., \& Gervais, F. 2002, J. Phys.: Condens. Matter, 14, 3543

Thibaudeau, P., Debernardi, A., Ta Phoc, V., da Roche, S., \& Gervais, F. 2006, Phys. Rev. B, 73, 064305

Thomas, M. E., Joseph, R. I., \& Tropf, W. J. 1998a, Appl. Opt., 27, 2

Thomas, M. E., Andersson, S. K., Sova, R. M., \& Joseph, R. I. 1998b, Infrared Phys. Techn., 39, 235

Tropf, W. J., \& Thomas, M. E. 1991, in Handbook of Optical Constants of Solids II, ed. E. D. Palik (Boston: Academic Press), 883 